\title{
Modeling of cytochrome P450 (Cyt P450, CYP) channels*
}

\author{
Christopher K. Jankowski a,b,d,**, Julien B. Chiasson ${ }^{\text {a,c }}$, Étienne Dako ${ }^{\text {a,d }}$, Kathy Doucet ${ }^{\text {a,b }}$, \\ Marc E. Surette ${ }^{\mathrm{a}, \mathrm{b}}$, Francois André ${ }^{\mathrm{a}, \mathrm{e}}$ and Marcel Delaforge ${ }^{\mathrm{a}, \mathrm{e}}$ \\ ${ }^{a}$ Faculté des études supérieures et de la recherche, Université de Moncton, Moncton, NB, Canada \\ ${ }^{\mathrm{b}}$ Faculté des Sciences, Département de chimie et biochimie, Université de Moncton, Moncton, NB, \\ Canada \\ ${ }^{\mathrm{c}}$ Département de l'informatique, Université de Moncton, Moncton, NB, Canada \\ ${ }^{\mathrm{d}}$ Faculté des sciences de la santé et des services communautaires, Université de Moncton, Moncton, \\ NB, Canada \\ ${ }^{\mathrm{e}}$ Centre d'études nucléaires de Saclay, CEA, iBiTec-S/SB2SM, URA2096 CNRS, Gif-sur-Yvette, France
}

Abstract. The precise location of a substrate in cytochrome P450 (CYP) governs the orientation of the oxidation position. Such information is generally obtained from biochemical data, but modeling approaches have also been used to explain these locations.

We used X-ray data and modeling techniques to distinguish between the series of putative linear or curved channels which lead the substrate from the outer side of the protein to the inner, and then into the heme pocket; these techniques were also used to identify the largest such channels. Two new methods for precisely determining the 3-D structure of proteins using $\mathrm{X}$-ray crystallography were proposed in order to identify these channels: first, the use of both straight and curved channels, and second, the sphere method. These data are compared with Poulos channels, and with Caver (or Mol on line) modeling methodologies.

Our methods were developed from studies of the interaction between cytochrome P450 CAM (CYP101) from Pseudomonas putida (as expressed in Escherichia coli) and the indolic base $\beta$-carboline. Apart from the identification of potential access channels leading to the heme-containing active site, a new explanation was advanced for the substrate's hydroxylation position. The sphere method seems to have potential to become a general and direct method for prediction of substrate access channels from reduced- or low-resolution crystallographic data.

Keywords: CP450 (CYP) channels, oxidation by CP450 (CYP), prediction of channels from reduced X-ray data

\section{Abbreviations}

$\begin{aligned} \text { P450, CP450 and CYP } & \text { cytochrome P450; } \\ \text { PDB } & \text { Protein Data Base; } \\ \text { cam } & \text { camphor; } \\ \text { Caver } & \text { MolAxis, Mol on line. }\end{aligned}$

\footnotetext{
${ }^{*}$ We would like to dedicate this study to the memory of Dr. Francisco Söler, professor at Université de Moncton.

${ }^{* *}$ Corresponding author: Christopher K. Jankowski, Département de chimie et biochimie, Université de Moncton, Moncton, NB, E1A 3 E9, Canada. Tel.: +1 506858 4331; Fax: +1 506858 4541; E-mail: jankowc@ umoncton.ca.
} 


\section{Introduction}

In our previous studies [3-6,14,22,33,47,48], we have determined that the oxidation of the indolic alkaloid $\beta$-carboline (1(R)-1,2,3,4-tetrahydro-1,2-butanopyridino[3,4-b]indole) (1) with cytochrome $\mathrm{P} 450$ (CYP) leads to three mono hydroxylated products: 4-hydroxy, 6-hydroxy and 7-hydroxy carbolines, with the major product being 6-hydroxy carboline. The position of hydroxylation was established by extensive deuteration studies, and by comparison to other oxidation models (such as Gif [3-6] and ferrous picolinate oxidations [47]) (also see Appendix 3). To explain these results, we have attempted a computer-assisted modeling study of the interaction between $\beta$-carboline and Cyt P450 (CYP), and performed a search for access channels leading to the heme's -Cys-containing pocket (Fig. 1).

In the abundant literature on this subject, the modeling of Cyt P450 (CYP) is essentially reduced to different transformations of X-ray data for bacterial cytochrome from Pseudomonas putida grown on (for example) camphor $[2,20,23,40,45,49]$. The present study used crystallographic data compiled by Poulos, as extracted from Brookhaven's Protein Data Bank [2,41-43]. Our first approximation was to reduce the data to the coordinates of the $\alpha$-carbon framework of the 414 amino acids from this protein, organized in $\alpha$-helices, $\beta$-sheets and $\beta$-turns, with a few remaining short chains uninvolved in the secondary structural hydrogen-bonding. We used only 405 of the $414 \alpha$-carbon X-ray coordinates; the nine $\mathrm{N}$-terminal amino acid residues, which float freely as a terminal chain, were disregarded. The coordinates for the heme were taken from the same crystallographic data file.

It is important to note that all $\alpha$-carbons and heavy atoms were taken into account. The hydrogen atoms and the water molecules were excluded from the Poulos PDB data for the Cyt P450 ${ }_{\mathrm{CAM}}$ (CYP101) used in this modeling (Fig. 2).

In the literature, these data have often been used to model systems other than cytochrome from bacteria grown on camphor. We assumed that the Cyt P450 (CYP) would adopt a generally similar structure when reacting with the $\beta$-carboline (1) substrate, following the practice of (if $\mathrm{X}$-ray data is unavailable) using model compounds as similar as possible to the one being studied. The protein frame of the Cyt P450 (CYP), which plays an important role in the orientation of the substrate toward the oxidation site and in substrate transport and extraction from the enzyme, should essentially remain unchanged. It
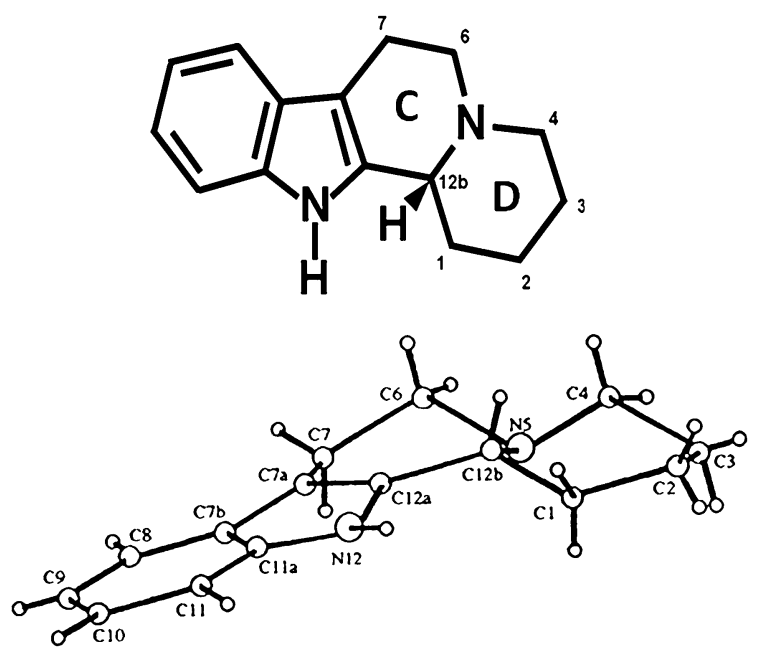

Fig. 1. Carboline (1). 


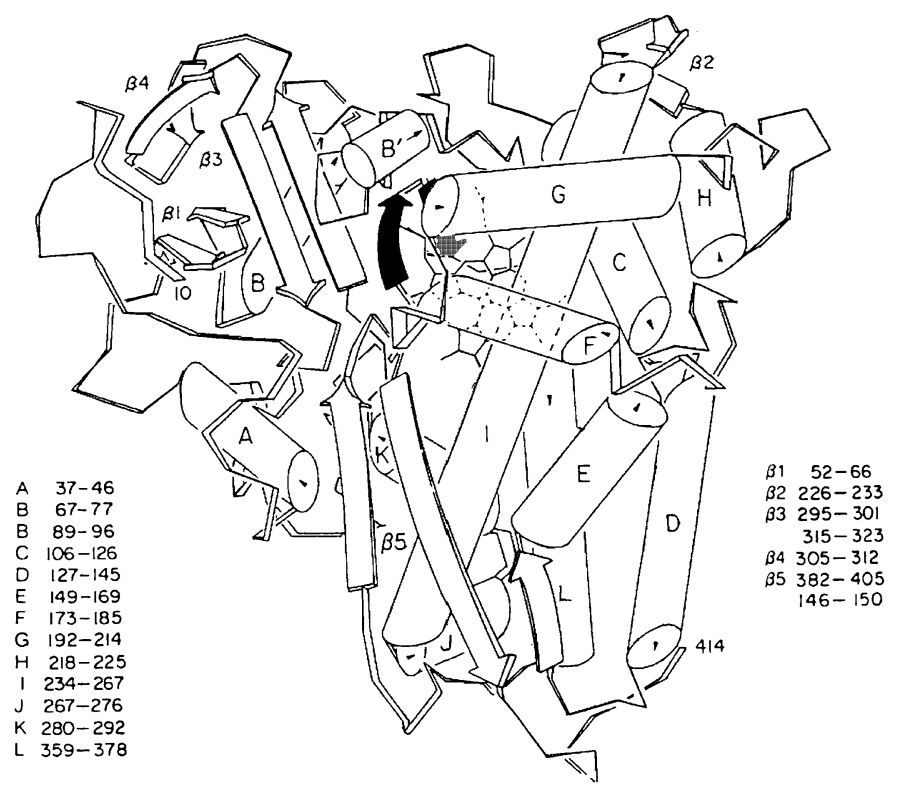

Fig. 2. CP450 (CYP101) adapted from Poulos [43].

is also assumed that, within a cytochrome family, a significant degree of primary-sequence homology is observed. For example, the use of mammalian Cyt P450 (CYP) (as opposed to the bacterial (P. putida) cytochrome camphor complex, whose coordinates are known from Poulos' work) is acceptable, because the frame of the cytochrome protein sub-units remains roughly the same despite some minor variations (primary sequence, limited rotations, thermal change, specific openings, etc.). It is also known that, both in bacterial and in some mammalian CYP, all type I substrates can be integrated into the active site close to the heme $[19,27,28,30,34-36,38,44,46]$.

\section{Oxidation of $\beta$-carboline (1) by cytochrome}

The fact that the carboline is oxidized in three activated positions (two of which are $\alpha$ to nitrogen, and one of which is benzylic) is not surprising, and can be related to a one-electron oxidation process independent of the Cyt P450 (CYP) oxidation pathway ( $\mathrm{N}_{5}$ or N-indolic oxidation). In the case studied, the enzymatic reaction carried out was rigorously similar to the model reaction (e.g., auto-oxidation), and the reported results are those of the Cyt P450 (CYP) oxidation process only. The examination of the three metabolites identified as a result of the oxidation showed that the substrate $\mathbf{1}$ is close to the heme when it enters the active site, presenting all $\mathrm{C}$ and $\mathrm{D}$ ring methylenes toward the heme; alternately, if the substrate enters the heme area differently, the three methylenes (C-4, C-5, C-7) pass through the plane perpendicular to the heme-dioxygen complex. In fact, modeling of this part of the Cyt P450 (CYP) shows that, in order to obtain their proper orientation, the Cyt P450 (CYP) should align all three methylenes (4, 6 and 7) along the same axis. Such an alignment facilitates the $\beta$-carboline's (1) passage through the vicinity of the heme, and explains the activation of these methylenes ${ }^{1}$ (Appendix 3 ).

\footnotetext{
${ }^{1}$ The "docking" of the substrate must be at least 2.6-3.0 ̊ from the heme, in order to be oxidized.
} 
Available X-ray data was used in developing a method for identifying channels which guide substrates to the oxidation heme pocket. After our preliminary modeling work in which we searched for less hindered channel pathways by slicing the protein into 1-Å slices (Appendix 1), two more advanced methods were developed, both of which are described in this paper: the straight and curved channels method (based on the Bézier tubes concept $[12,13,25]$ ) and the sphere method.

These methods were also compared to the existing methods for predicting substrate access channels, such as Caver's Mol on line [8], with constant comparison to two channels observed by Poulos as a result of biochemical considerations [7,11,21,39,41-43,50,51].

It is also assumed that the identification of such channels will facilitate the study of water-soluble P450 for which, as reported in the literature [10] for membrane P450, reductase docking enabled us to deduce the pre-orientation of such channels.

The test substrate, carboline $\mathbf{1}$, was simplified for these computations to a rectangular box of dimensions of approximately $2 \AA \times 4 \AA \times 8 \AA$. This box's passage through the channel identified from the modeling enabled us to decide whether or not a channel could potentially be the channel leading to the heme; the passage is therefore a decisive criterion for selection.

The objective of this paper is also to elaborate a more efficient a priori methodology for determining potential channels, to be able to apply this methodology to other enzymes, and to offer a general methodology of channel prediction from X-ray data.

In this work, the possibility of finding straight or curved channels will be explored, as will the search for the largest allowable diameter of those channels.

\section{Determination of potential access channels}

\subsection{Straight and curved channels}

Unlike the previous method used by our group, in which the Cyt P450 (CYP) was cut into 1- $\AA$-thick slices, this approach analyzed potential channels (represented by tubes of given diameter) from a virtual vantage point on the iron of the heme, where substrate-compatible pathways are more easily recognizable (Appendix 1).

Numerous tubes were projected from the heme iron to the exterior of the Cyt P450 (CYP), in order to identify the passages of lesser atomic density; these passages would be less hindered, and therefore more suitable for the passage of the carboline substrate (Fig. 3).

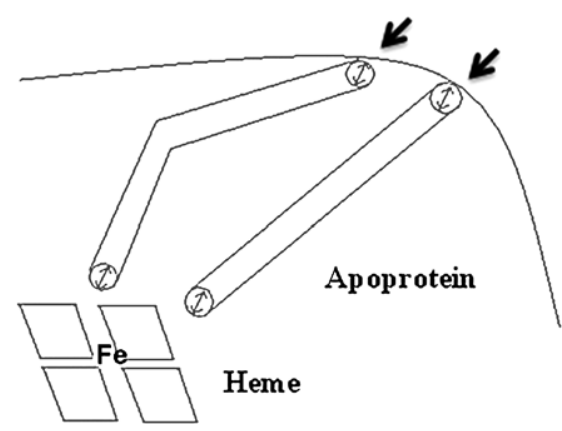

Fig. 3. Curved (upper) and straight (lower) channels providing access to the heme. 


\subsection{Substrate entry indicated by arrows}

The search for straight and curved channels involves compiling a congestion chart - that is, a hindrance map for the transport of substrate. This consists of three parts: the first two involve the search for straight channels by the determination of a congestion chart (hindrance map with the appropriate indexes and a channel radii mapping-tube radius), and the third involves the search for curved channels. An algorithm has been developed to establish those three methods using the aforementioned congestion chart [12,25].

\subsubsection{Congestion chart and channel radii mapping}

The congestion indexes are created by considering the approximate radius of the tubes starting from the heme centre (iron), and the size of one of the six sides of the enzyme considered as a box. This chart enables us to visualize the potential zones of passage of the substrate, thereby allowing the formation of straight tubes in these regions. In order to determine these regions, different vectors have been constructed in several directions starting from the iron. ${ }^{2}$

For each such direction, a vector perpendicular to the surrounding atoms is created. The number of atoms is counted for each tube of a given radius and direction between the iron and the exterior of the molecule. This atom count permits us to determine the density - i.e., the number of non-hydrogen atoms able to obstruct the substrate's passage through the tube. A chart of hindrance of passage - a "congestion index" - was then created to list those different densities (Fig. 4).

There are six sections of the chart, each representing part of the protein: the top, bottom, front, back, right and left. This chart shows the number of atoms obstructing the passage through the tube for each point: the smaller numbers represent passage zones which are less obstructed. Figure 5 shows an estimated chart of congestion indexes for the bottom section of the protein. Examination of this figure reveals a large zone (outlined in red) where these index numbers are smaller, from 7 to 9; this indicates a potential passage zone for substrates of given dimension (for example for alkaloid 1) (Fig. 5).

\subsubsection{Mapping of channels (tubes) radii}

A chart was created, listing channels by their radii in Ångstroms. A larger radius means a larger channel, which could better allow passage of the substrate.

This chart was used to search for straight tubes. As with the previously-described method, millions of vectors were projected in different directions from the iron. For each direction, the perpendicular distance between the atoms and the vector was determined. Afterwards, the minimal distance for the atoms

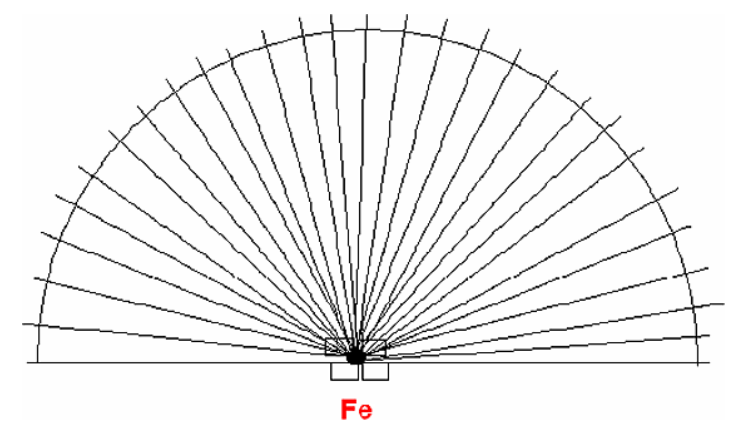

Fig. 4. Projection of vectors starting from the heme iron. (Colors are visible in the online version of the article; http://dx.doi.org/ 10.3233/SPE-2011-0499.)

\footnotetext{
${ }^{2}$ This congestion chart data have been computed with the use of the $x, y$ and $z$ axes as defined by the authors.
} 


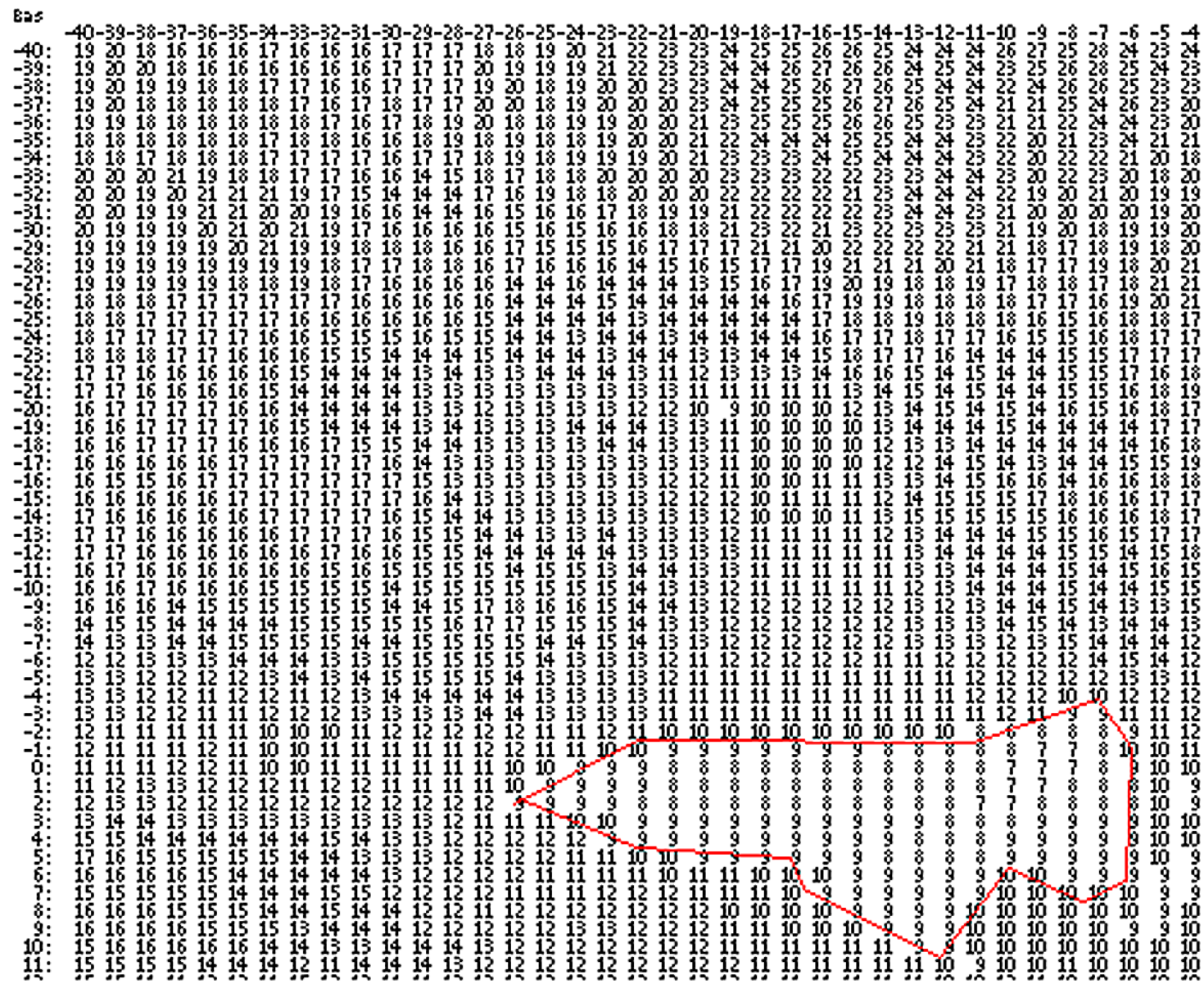

Fig. 5. Map of a congestion index (bottom part of the protein 1). The centre of lowest congestion for this cut (at 7) is estimated as being on a plane $-15 \AA$ (horizontal upper scale) and $+2 \AA$ (vertical left scale) from the heme; $\mathrm{Fe}(0,0,0)$ is centre of the coordinates (Origin). The corresponding channel exit is situated near helices F and I. (The colors are visible in the online version of the article; http://dx.doi.org/10.3233/SPE-2011-0499.)

between the iron and the exterior of the molecule in the direction indicated was likewise determined. This distance enabled us to approximate the radius of a tube containing no atoms. The radii for each direction were computed, and a complete chart of tube radii was created (Fig. 6).

In this chart, the channel radii (tubes) were numbered from $0-9$, with the numbers $0,1,2,3,4,5,6$, 7,8 and 9 representing, respectively, radii between $(0.0-0.2),(0.2-0.4),(0.4-0.6),(0.6-0.8),(0.8-1.0)$, $(1.0-1.2),(1.2-1.4),(1.4-1.6),(1.6-1.8)$ and finally (1.8-infinity) Ångstroms. By examining the chart of channel radii (Fig. 6), it was possible to detect an interesting zone (outlined in red), where the bigger numbers $(6,7$ and 8 ) indicated tubes of particularly large radii; this zone should be considered as a potential channel for the substrate.

\subsubsection{Search for curved channels. Curved-channel methodology}

A second method developed to locate channels was the use of curved tubes. In this method, for each chosen vector originating from the iron atom, several points were calculated at every $0.5 \AA$ along this vector. For each point, the closest atom was determined. If the distance from this atom was smaller than 


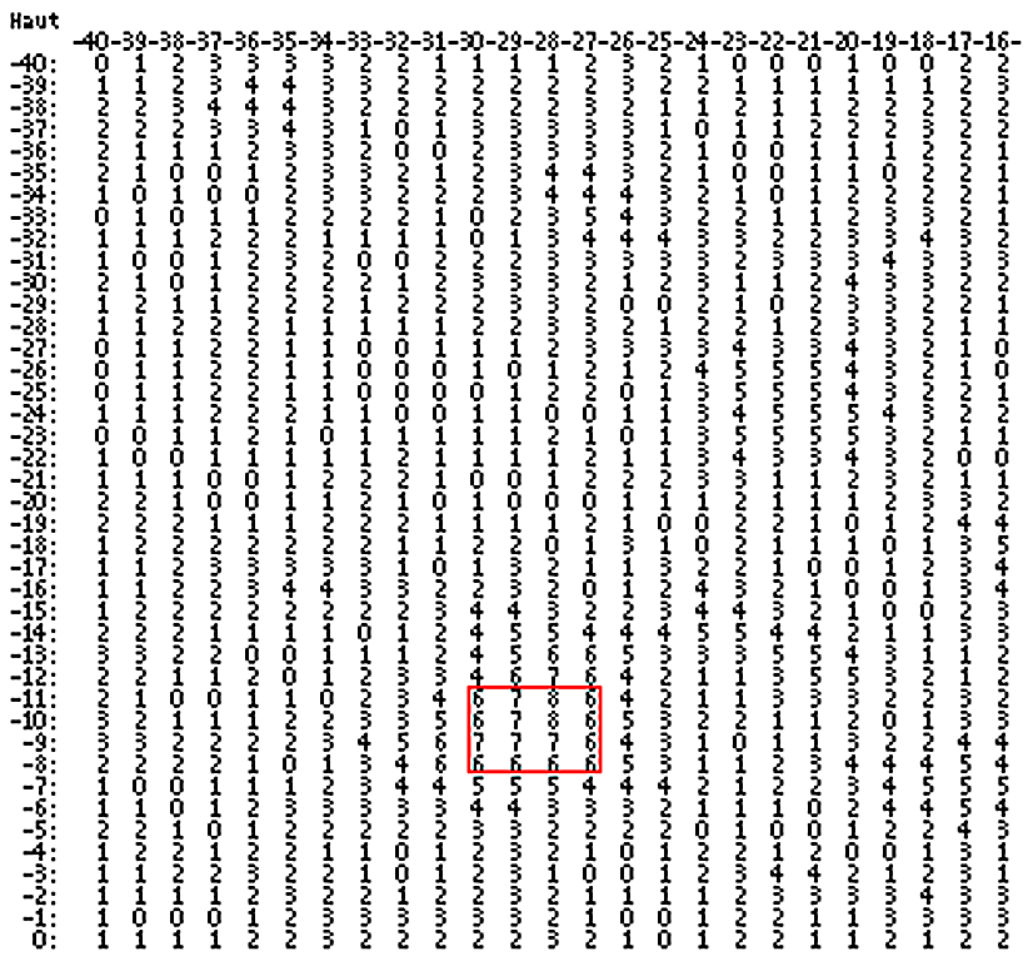

Fig. 6. Chart of tube radii (bottom part of the protein 1). The tube of largest radius, at $7 \AA$, is situated at the intersection of planes $-29 \AA$ (upper scale) and $-9 \AA$ (left side scale); Fe atom is $(0,0,0)$ and the heme is in the horizontal plane. The exit of the corresponding channel is situated near Tyr 179 and Asp 245 (between helices F and I). (The colors are visible in the online version of the article; http://dx.doi.org/10.3233/SPE-2011-0499.)

the desired radius for the tube (for example, this particular substrate required a radius of ca $2.25 \AA$ ), then the point was moved radially (relative to the tube) away from the atom so as to find the largest distance between the new point and its closest atom. Note that the closest atom's origin may change whenever a point is moved, which means that it does not necessarily belong to the same helix or $\beta$-sheet amino acid. The radius of the curved tube was calculated as the minimal distance between these new points and their closest atoms.

Figure 7 shows a sample of the table created during the search for curved tubes. This table represents the data obtained for the tube generated in the direction $(-15,40,40)$ for the $x, y$ and $z$ axes, respectively. The points from 0 to 30 and their coordinates were referred to these three axes; the columns of this table show the coordinates, the distance of the closest atom from the point, and the number of that atom according to the PDB. With that number, it was possible to determine to which amino acid of the cytochrome body the atom belongs. Finally, the last column represents the distance between the point and the origin (the iron).

In this example we have only considered the portion of the tube starting at $3 \AA$ from the iron in the direction of the molecule's outer surface (exit of channel). The result ( $2.44 \AA$, outlined in red) is the estimated distance to the closest atom, which represents the radius of the curved tube. This tube is very promising, since it could allow the passage of the test substrate (carboline) which has a smaller radius of only $2.25 \AA$. 
Tube orientation: $(-15,40,40)$

Fe: $(\mathbf{0}, \mathbf{0}, \mathbf{0})$

Number of Iterations: $\mathbf{5 0 0}$

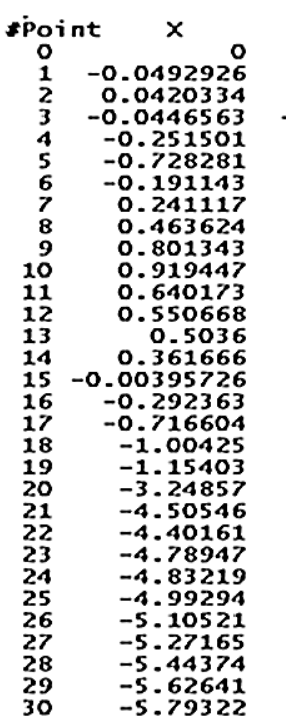

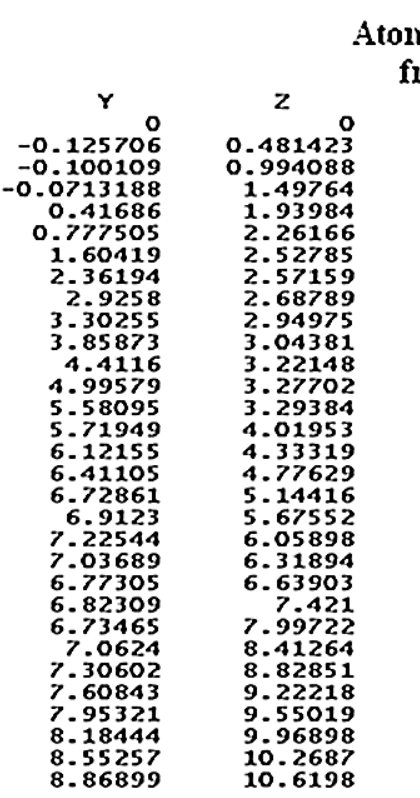

\begin{tabular}{|c|c|}
\hline $\begin{array}{l}\text { tom distance } \\
\text { from } \mathrm{Fe}\end{array}$ & $\begin{array}{l}\text { Neighbour } \\
\text { atom number }\end{array}$ \\
\hline $\begin{array}{l}1.97168 \\
1.96015 \\
2.13333 \\
2.37228 \\
2.47714 \\
2.59111 \\
2.44899 \\
2.43522 \\
2.50656 \\
2.64952 \\
2.86188 \\
2.89289 \\
3.1079 \\
3.23619 \\
3.23136 \\
2.92687 \\
2.73189 \\
2.59307 \\
2.62369 \\
2.63938 \\
2.44518 \\
2.57055 \\
2.63941 \\
2.69353 \\
2.74307 \\
2.69033 \\
2.61767 \\
2.55626 \\
2.62777 \\
2.79067 \\
3.01121\end{array}$ & $\begin{array}{r}4 \\
4 \\
2 \\
4 \\
3211 \\
4 \\
1878 \\
3220 \\
3177 \\
3220 \\
3178 \\
1878 \\
2235 \\
3181 \\
1873 \\
2227 \\
2227 \\
1875 \\
1875 \\
1897 \\
1875 \\
1866 \\
1900 \\
1890 \\
1870 \\
1891 \\
1312 \\
1899 \\
1312 \\
1899 \\
1899\end{array}$ \\
\hline
\end{tabular}

Neiglbour atom
distance from Fe

Fig. 7. Search for curved channels. The area identified as 2.44518 corresponded roughly to Poulos' main channel. (The colors are visible in the online version of the article; http://dx.doi.org/10.3233/SPE-2011-0499.)

\subsubsection{Curved channels}

The search for curved channels was performed by creating a channel one point at a time, instead of finding a pre-existing potential channel and modifying it. This modification uses a recursive algorithm to create density charts at $1 \AA$ steps, and then calculates them from the heme to the exterior of the protein. This enabled us to locate potentially less-hindered channels, which are suitable for the passage of the substrate. Starting from the iron, vectors were created in 26 different directions (because there are $26 \mathrm{C}_{\alpha}$-points close to the iron), along the $x, y$ and $z$ axes. Vectors were extended incrementally ("steps") until they were obstructed by one or many atoms. Obstructed vectors were deviated at the obstruction point until they were able to continue; deviations which led inward again were excluded. We assume that a single channel is formed of $M$ points: [Si], $i=0$ to $M-1$. As a first approach, the [Si] will only be able to move on a sphere with the iron as the origin. $M$ is chosen so that $S[M]$ is situated on the exterior of the molecule. A channel is considered to have been completed if it reaches a point $S[M]$ on the exterior of the molecule.

Using the coordinates of the straight channels established earlier, the length of the channel was determined. This involved a simple calculation using the original coordinates of the iron and of the channel in question. The angle of the channel and its position in the space of the protein can be determined with those coordinates. Figure 8 shows an example of a straight channel in the protein's space, where $L_{1}$ represents its length and $\theta_{1}$ represents its angle formed with the origin. The method used to calculate the length of the curved channels is similar to that used for the straight channels. For two straight channels, their respective lengths were estimated individually and their angles were calculated from the angles of two straight channels. Figure 9 presents an example of a curved channel in the protein's space: $L_{1}$ represents the first straight channel starting from the origin, $L_{2}$ the second straight channel starting from the origin, $\theta_{1}$ the angle of the first channel relative to the origin and $\theta_{2}$ the angle of the second channel axis 


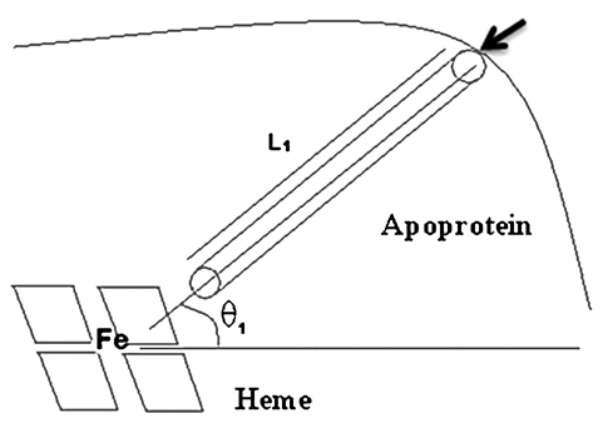

Fig. 8. Straight channel. Arrow indicates the substrate entry, $\theta_{1}$ the inclination angle $L_{1}$ the length of channel.

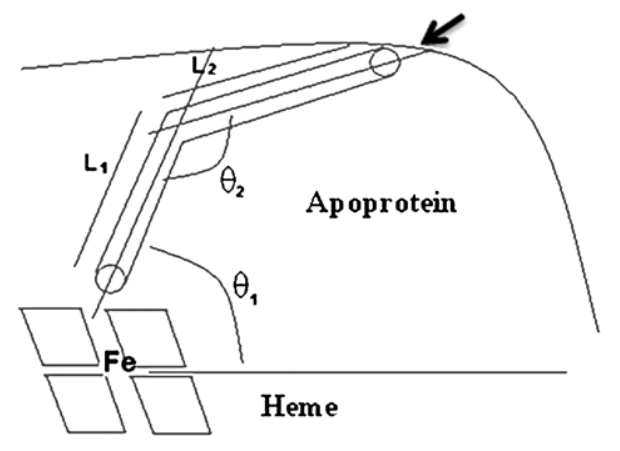

Fig. 9. Curved channel. Arrow indicates the substrate entry, $\theta_{1}, \theta_{2}$ the inclination angles, $L_{1}, L_{2}$ length of two linked channels.

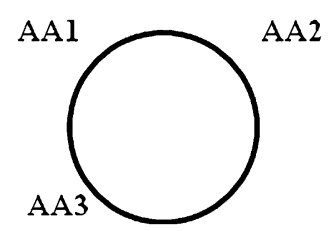

Fig. 10. Identification of a channel entry with help of three nearest amino acids (AA). Using a three-AA nomenclature, the main Poulos channel is named Thr 185 (helix F), Phe 87 (helix B), Ile 395 ( $\beta 5$ ).

relative to the first channel axis, respectively. The identification of the exact position of the straight and curved channels is therefore possible.

It is important to precisely locate the straight and curved channels once they have been identified. This will be done with the help of the coordinates of the $\alpha$-carbons of three amino acids situated at the channel entry, at the outer surface (exterior) of the protein. Figure 10 shows an example of a transversal cut of a channel with three different amino acids contouring it, which would be chosen for its unambiguous identification.

\subsection{Preliminary evaluation of the results}

The results obtained from these three variant methods for identifying access channels allowed us to select the "best" substrate-compatible channel(s) from the "good choice" group of channels. With the help of the chart of tube radii, one straight channel with a radius of $1.6 \AA$ was found. This channel was identified by three amino acids located at its entry; although this channel did not allow the direct passage of the carboline, it is a preliminary indication of a potential zone which could be examined with the other methods (for instance, curved channels). These tubes, which will easily allow the passage of the carboline, were not the same as the two previously identified by Poulos or by the "slicing" method. This problem was approached using both the curved channels method and the spheres method (see Section 3.4).

The curved channels method can be considered promising because a straight channel can be obstructed by atoms, whereas a curved channel can deviate out of the way. The curved channel identified had a radius of $2.44 \AA$ - sufficient to allow the passage of the carboline. The entry to this curved channel was identified and located on the exterior of the protein surface (see Fig. 10 legend). 
To explain the differences between the predicted channel structures, their orientations as observed by Poulos, and the results obtained via newer protein-modeling programs (such as Caver or MolAxis), the sphere method was developed as described in Section 3.4.

\subsection{Sphere method}

As with previous calculations, the atom coordinates of the CP450 necessary for this computation were extracted from the file 1AKD.pdb (source: pdb.org) [2], and the coordinates of the atoms of the camphor and water were removed from the data set. For the purpose of this study, the heme cavity was approximated to an empty sphere within the protein. The radius of that sphere is set as the distance between the center of the sphere and the closest CP450-Cyt non-hydrogen atom.

The centre of such a defined cavity, previously occupied by the camphor, was used as the endpoint for all access paths. A simple grid search was designed to find the position and diameter of the largest cavity in that area.

The topology of CP450-Cyt was transformed into a series of concentric layers of spherical cavities. To compute a layer of cavities, points were generated on a sphere centered on the centre of the central cavity. For each of these points, a cavity was computed. If the radius of the cavity was larger than a given threshold, this particular cavity was kept for further considerations; those which were too small to allow the passage of a given substrate were discarded.

Some constraints were applied to the generation of the aforementioned layers. The distance between two neighbouring points in a layer was made small enough to find reasonably good cavities in any area (assuming such cavities did exist). The distance between two layers was made sufficiently small to ensure that it would be possible to have reasonably large intersections between cavities from neighbouring layers, so as to let the substrate molecule through. The last layer was set to be far enough from the centre - that is, completely outside the cytochrome body - for there to exist a complete passage from the exterior surface of the protein to the central cavity. The details of the algorithm developed are presented in Appendix 2.

To simplify the calculations for paths from the center of the substrate to the exterior, all dead ends were deleted. The central cavity layer was numbered 0 . In the next layer (layer 1), all cavities without sufficiently large connections to the cavity in layer 0 were removed. Similarly, all layer- 2 cavities were removed unless they had a sufficiently large connection to at least one cavity in layer 1 . This method was applied to all succeeding layers until the outer layer, layer $N$, was reached. This allowed us to eliminate all center-oriented dead ends.

To remove the exterior-oriented dead-ends, we began at the outer layer $N$ and proceeded inward. In layer $N-1$, all cavities without sufficiently large connections to at least one cavity in layer $N$ were removed; in layer $N-2$, all cavities without sufficiently large connections to at least one cavity in layer $N-1$ were removed, etc.

Once all the dead ends are removed, finding a pathway from layer 0 to layer $N$ becomes relatively straightforward: it can be defined as a series of interconnecting cavities from layer to layer. However, finding the best path is computationally daunting, because the number of possible paths can be exponential to the number of layers. Therefore, instead of finding the overall "best" path, a heuristic was used to find a set of "reasonably good" paths. The search for a "good" path was begun in a middle-layer cavity. From this point, two half-paths were found: one going towards the central cavity, and one going towards the outer layer. The sum of these two half-paths makes a complete path between the central cavity and the outer cavity (Fig. 11). 


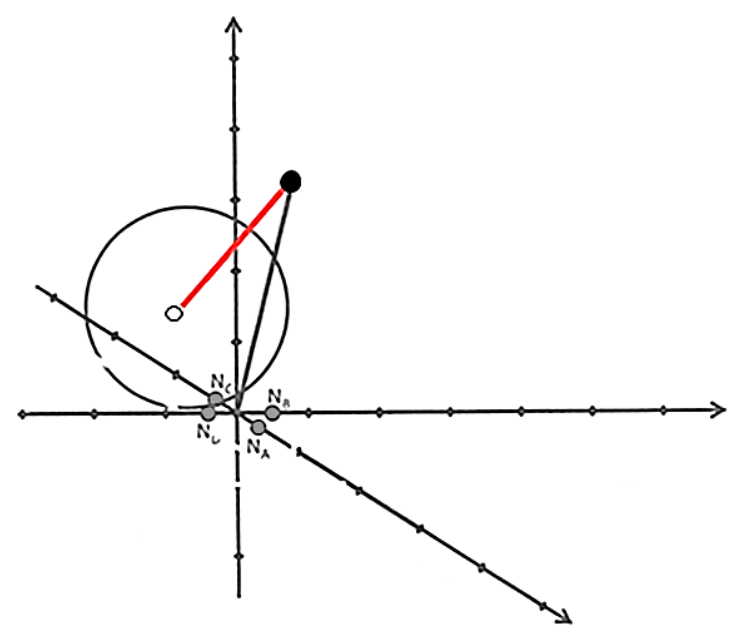

Fig. 11. Example of channel. Channel \#392: cavity centre coordinates (open circle point): $3.1,-4.1,7.6$; cavity radius $=7.1 \AA$ (in red). First sphere coordinates: 8.7, -4.2, 20.9 (black point). Distance to Fe: $2.3 \AA$, average channel \#392 radius $4.8 \AA$. $N_{\mathrm{A}}-N_{\mathrm{D}}$ four pyrrole nitrogens of heme, $\mathrm{Fe}$ is on the Origin of coordinate. The intersection is built from the first sphere to the next one, etc. (The colors are visible in the online version of the article; http://dx.doi.org/10.3233/SPE-2011-0499.)

For a given cavity, to find the best intersecting cavity into the next layer, the intersections between that cavity and all the cavities in the next neighbouring layer were computed. The neighbouring cavity with the largest intersection was identified and retained as the next step in channel path determination.

This operation was repeated for each layer, from one best intersecting cavity to the next, until the central cavity was reached. By searching inward to the central cavity and outward to the outer layer, the cost of finding a path is now linear to the number of cavities in the protein body. A set of good paths was defined by finding, for each cavity in a middle layer, a path between the central cavity and the outer layer. Thus, the cost of finding a set of paths is roughly equal to the total number of cavities in the protein multiplied by the number of cavities in the middle layer: there will be as many paths as there are cavities in the chosen middle layer. The set of good paths was then subdivided according to the exit points. For each subset, the best path could be chosen. The 'best' path is the one with the largest radius, allowing the easiest passage of the substrate. This path is then represented as a sequence of partially overlapping spheres; it was concluded that this set of paths represented the best overall path.

As was mentioned at the beginning of this section, two sets of computations were performed: one which used all the $\mathrm{Fe}, \mathrm{C}, \mathrm{N}$ and $\mathrm{O}$ atoms of the $\mathrm{CP} 450$; and one which used only the $\mathrm{Fe}, \mathrm{C}, \mathrm{N}$ and $\mathrm{O}$ atoms of the heme, and the $\alpha$ carbon atoms of the amino acids. The first set is probably too restrictive: it considers the protein as a rigid crystallized entity, without any flexibility. The second set is probably too flexible; however, its advantage is that it simulates the ability of the amino acids to move out of the way of the incoming small substrate molecule.

As expected, the size of the paths found by the two methods differed significantly. For the flexible, allatom framework, the best of the good paths had a radius of about $2 \AA$, while for the rigid, limited-atom framework, the best of the good paths had a radius of about 4.1-4.8 $\AA$. In practice, it can be assumed that the true value of the radius is somewhere between those two extremes.

Using this method, seven channels were identified as being large enough to allow the carboline substrate passage from the outside of the protein to the heme oxidation site; after careful consideration, two of the channels, E and G, were eliminated (Table 1, Figs 12 and 13). 
Table 1

Seventeen "best" channels identified by the sphere method (A-G)

\begin{tabular}{|c|c|c|c|c|c|c|c|c|c|c|c|c|c|c|c|c|}
\hline & \multirow[t]{2}{*}{ Path no. } & \multicolumn{3}{|c|}{ Path end coordinates } & \multirow[t]{2}{*}{$\alpha$} & \multirow[t]{2}{*}{$\gamma$} & \multirow{2}{*}{$\begin{array}{c}\text { dist-Fe } \\
(\AA)\end{array}$} & \multirow{2}{*}{$\begin{array}{l}\text { Length } \\
(\AA)\end{array}$} & \multirow{2}{*}{$\begin{array}{c}r \\
(\AA)\end{array}$} & \multirow[t]{2}{*}{ Atom } & \multirow{2}{*}{$\begin{array}{c}\text { distCavit } \\
(\AA)\end{array}$} & \multirow[t]{2}{*}{ AA } & \multicolumn{3}{|c|}{ AA end coordinates } & \multirow{2}{*}{$\begin{array}{l}\text { Length/ } \\
\text { distCavit }\end{array}$} \\
\hline & & $x$ & $y$ & $z$ & & & & & & & & & $x$ & $y$ & $z$ & \\
\hline $\bar{A}$ & 3793 & 6.20 & 19.57 & 5.98 & 72.43 & 16.24 & 21.39 & 31.90 & 4.10 & 227 & 23.93 & Gln 39 & 8.80 & 14.66 & 7.88 & 1.33 \\
\hline B & 2579 & 23.42 & 13.28 & 14.26 & 29.56 & 27.91 & 30.47 & 47.18 & 4.42 & 110 & 27.53 & Phe 24 & 23.85 & 8.23 & 14.64 & 1.71 \\
\hline $\mathrm{C}$ & 18 & -15.35 & -8.74 & 11.93 & -150.34 & 34.04 & 21.33 & 25.09 & 4.09 & 1310 & 19.53 & Asp 173 & -16.58 & -3.74 & 13.90 & 1.28 \\
\hline \multirow[t]{3}{*}{$\mathrm{D}(\mathrm{G})$} & 3502 & -6.30 & 5.51 & 14.21 & 138.85 & 59.49 & 16.50 & 18.69 & 4.08 & 1351 & 14.98 & Lys 178 & -8.74 & 0.02 & 13.87 & 1.25 \\
\hline & 3310 & -6.91 & 5.66 & 15.44 & 140.70 & 59.94 & 17.85 & 22.59 & 4.08 & 1351 & 16.03 & Lys 178 & -8.74 & 0.02 & 13.87 & 1.41 \\
\hline & 3378 & -6.31 & 5.13 & 14.72 & 140.91 & 61.05 & 16.82 & 19.59 & 4.08 & 1351 & 14.98 & Lys 178 & -8.74 & 0.02 & 13.87 & 1.31 \\
\hline \multirow[t]{2}{*}{$\mathrm{F}(\mathrm{E})$} & 5 & 8.63 & -4.22 & 21.24 & -26.10 & 65.66 & 23.32 & 17.33 & 4.21 & 1463 & 14.68 & Thr 192 & 3.28 & -6.79 & 21.11 & 1.18 \\
\hline & 1 & 8.50 & -4.28 & 21.61 & -26.75 & 66.21 & 23.62 & 17.38 & 4.02 & 1463 & 14.98 & Thr 192 & 3.28 & -6.79 & 21.11 & 1.16 \\
\hline \multirow[t]{4}{*}{$\mathrm{E}(\mathrm{F})$} & 653 & 8.27 & -2.89 & 20.68 & -19.27 & 67.03 & 22.47 & 15.64 & 4.79 & 642 & 14.08 & Pro 89 & 12.25 & -4.05 & 15.95 & 1.11 \\
\hline & 956 & 8.21 & -2.63 & 20.68 & -17.77 & 67.37 & 22.41 & 19.42 & 4.79 & 642 & 14.08 & Pro 89 & 12.25 & -4.05 & 15.95 & 1.38 \\
\hline & 392 & 8.74 & -4.17 & 20.87 & -25.49 & 35.10 & 23.01 & 16.18 & 4.79 & 642 & 14.38 & Pro 89 & 12.25 & -4.05 & 15.95 & 1.13 \\
\hline & 652 & 8.69 & -3.92 & 20.89 & -24.30 & 65.46 & 22.97 & 16.40 & 4.79 & 642 & 14.38 & Pro 89 & 12.25 & -4.05 & 15.95 & 1.14 \\
\hline \multirow[t]{5}{*}{$\mathrm{G}(\mathrm{D})$} & 3478 & -2.08 & 5.23 & 18.13 & 111.74 & 72.74 & 19.00 & 20.00 & 4.08 & 1387 & 14.98 & Asp 182 & -2.59 & 0.80 & 14.94 & 1.34 \\
\hline & 3419 & -1.91 & 4.66 & 18.70 & 112.27 & 74.91 & 19.37 & 20.94 & 4.08 & 1387 & 14.98 & Asp 182 & -2.59 & 0.80 & 14.94 & 1.40 \\
\hline & 3340 & -1.92 & 4.45 & 18.85 & 113.31 & 75.56 & 19.47 & 20.48 & 4.08 & 1387 & 14.98 & Asp 182 & -2.59 & 0.80 & 14.94 & 1.37 \\
\hline & 3307 & -2.21 & 3.76 & 19.22 & 120.45 & 77.20 & 19.71 & 20.82 & 4.08 & 1387 & 14.98 & Asp 182 & -2.59 & 0.80 & 14.94 & 1.39 \\
\hline & 3301 & -2.22 & 3.32 & 19.50 & 123.80 & 78.40 & 19.91 & 21.19 & 4.08 & 1437 & 14.98 & Asp 188 & 1.71 & 2.14 & 22.63 & 1.41 \\
\hline
\end{tabular}

Notes: $\gamma$ - elevation angle with respect to the heme plane. Positive angles are above the plane, negative angles are below the plane; $\alpha-$ rotation angle with respect to an axis perpendicular to the heme plane and going through the Fe atom. Angle 0 is aligned with the Fe-NA direction; dist-Fe - the distance between the Fe atom and the exit point of the path $(\AA)$; length - the length of the path as approximated by the sum of the center-to-center distance between all the spheres making the path $(\AA) ; r$ - the radius of the path $(\AA)$. In this table, only the heme atoms and the $\alpha$ carbons were used, which explain the rather large radii; atom - the closest atom to the exit point of the path. It is the $\alpha$ carbon of the amino acid (AA) in the AA column; distCavit - the distance between the path end coordinates and the center of the central cavity; AA - the amino acid closest to the path end coordinates; length/distCavit - the ratio gives an indication of how straight or winding the path is. For example a ratio of 1.0 would indicate a straight path. A larger value would indicate how curved any given channel is. 


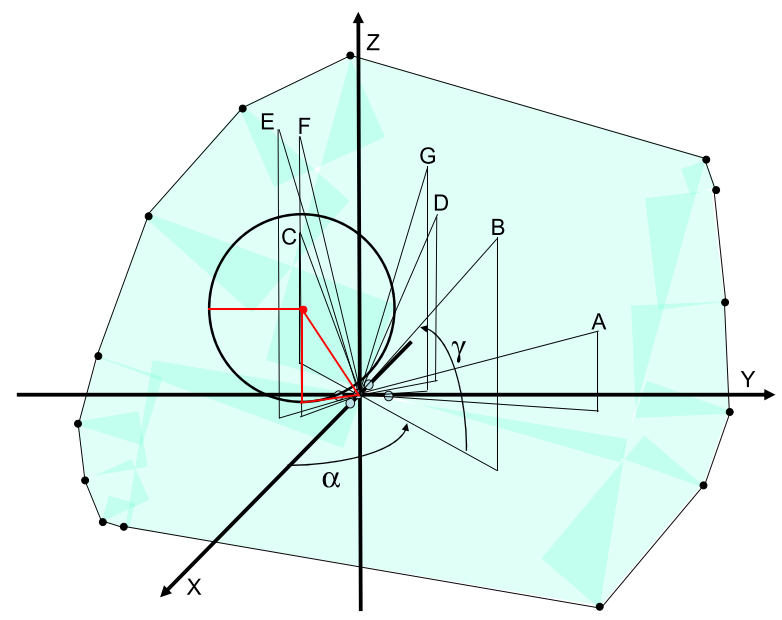

Fig. 12. Space orientation of seven channels (A-G). (Colors are visible in the online version of the article; http://dx.doi.org/ 10.3233/SPE-2011-0499.)
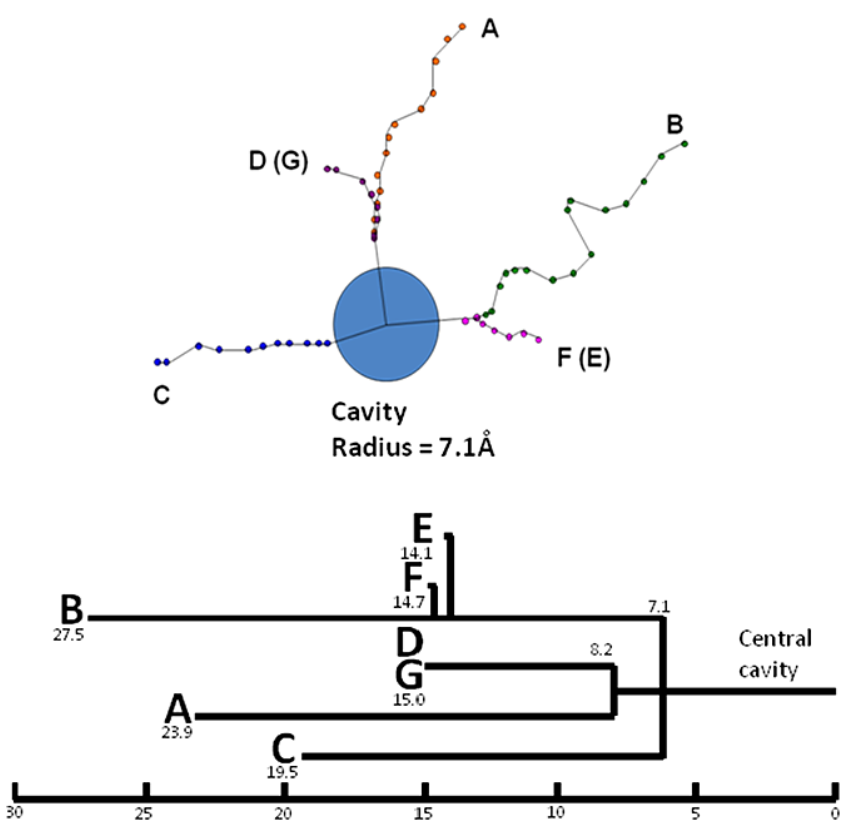

Fig. 13. Seven channels (A-G) identified by this method (upper graph); Dendrogramme of channels A-G, distance from cavity center to channel junctions (A) (lower graph). (Colors are visible in the online version of the article; http://dx.doi.org/10.3233/ SPE-2011-0499.)

The five remaining channels provide access to the heme through three very different routes. Although channels $A$ and $D(G)$ begin at very different parts of the outer envelope, and have different lengths (A begins at Gln 39 and is $23.9 \AA$; D begins at Lys 178 and is $15 \AA$ ), they have the same second half. $\mathrm{B}$ and $\mathrm{F}(\mathrm{E})$ likewise share a first part, although this common section is considerably shorter.

The $\mathrm{B}$ channel is the longest ( $47 \AA$ ), and $\mathrm{E}$ is the shortest (15 $\AA$, beginning at Pro 89). The $\mathrm{C}$ channel, beginning at Asp 172, has a length of approximately $25 \AA$. 
For the substrate transport to be effective, the channel radius must be greater than $4 \AA$. In this respect the channel $\mathrm{E}$ is the largest, followed by $\mathrm{B}$.

The effective length of substrate transport pathway is measured from the outer surface of the cytochrome to the center of the cavity, approximated to a sphere.

Five channels enabling carboline transport were predicted as the best results among 17; the best calculations are presented in Table 1.

Full descriptions of these channels are provided in Table 1 (see legend to this table): coordinates of the end of the path leading from the heme's central $\mathrm{Fe}$ atom to the channel entry on the outer envelope of the protein (Cartesian and spherical coordinates); the channel length; the minimal radius (in $\AA$ ) of the channel; the direct distance between Fe and the entry to the channel; and the amino acid closest to the channel entry and its coordinates. The last column presents the ratio between the actual distance traveled by the substrate and the direct distance, as an evaluation of these results.

Table 1 indicates that the seven possibilities for substrate entry are within proximity to Gln 39, Phe 24, Asp 173, Lys 178, Pro 89, Thr 192 and Asp 182 (which is also close to Asp 188). The full description of the pathway, as a function of the nearby helices and $\beta$-sheets segments of the protein, is also presented in the legend.

Comparison of the channels identified by our sphere method, to the two (or three) channels identified by Poulos for the cytochrome P450-CAM crystal structure [7,11,21,39,41-43,50,51] (A, B and C entries of substrate from the outside of heme side of the protein is close to Gln 400, Lys 126 and Arg 161, respectively), showed that all of our channels are among them. In addition, one Poulos channel was identified by our slice method (Appendix 1).

In order to pursue this comparison, the channels were identified by Caver-MolAxis methodology $[7,11,21,39,50,51]$ using the same crystal structure. Here, ten channels were identified which could be classified into five following groups (Table 2, Fig. 14): three groups from the distal side of the protein (total of seven channels), and two from the proximal side (three remaining channels).

All these distal side channels are relatively narrow; in order to allow carboline as a substrate, it will be necessary to use their dynamic properties. However, on the proximal side they are wider (Fig. 15).

The comparison of these results to ours allowed us to conclude the following.

1. Distal channel A, with the entry of substrate close to Gly 216 and Gln 227, does not correspond to any Caver-identified channels. Its characteristics are its excessive length ( $32 \AA$ ), and the large opening between the $\alpha$ carbons involved in its construction.

2. The two remaining distal channels $D$ and $G$ identified in our work are identical to Caver's group 1 and 2 channels, and followed almost identical pathways.

Table 2

Caver's identified channels (number 1-10)

\begin{tabular}{llc}
\hline Channels number & \multicolumn{1}{c}{ Substrate entry close to AA } & Opening size $(\AA)$ \\
\hline Distal side & & \\
1 and 2 & Gln 400 (and Asp 188) & 1.5 \\
$3,4,5$ (7) and 6 & Thr 192 (and Gly 189 and Glu 91) & 1.0 \\
9 & Ala 95 and Ala 194 & 1.2 \\
Proximal side & & \\
$\quad 6$ and 8 & Gly 226, Gln 117 and 227 & 1.5 \\
10 & Gln 117 & 1.1 \\
\hline
\end{tabular}




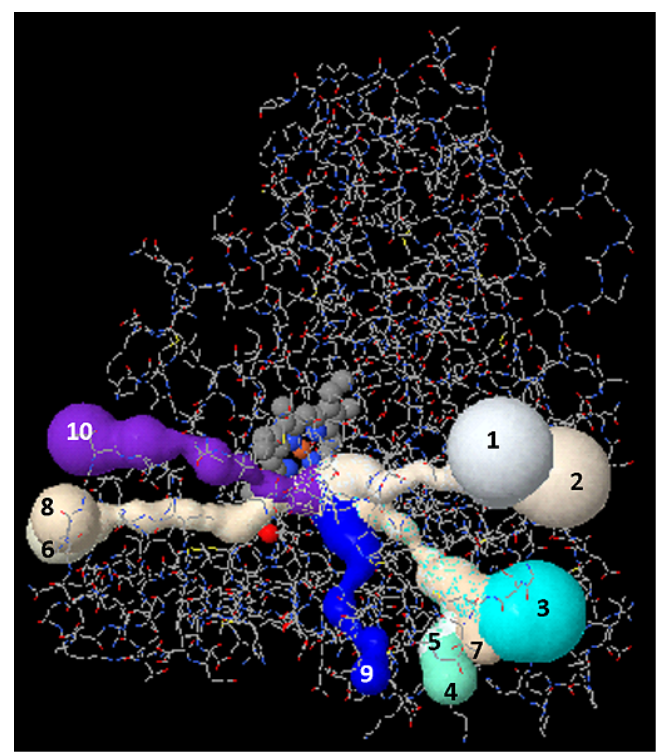

Fig. 14. Ten channels identified by Caver. (Colors are visible in the online version of the article; http://dx.doi.org/10.3233/SPE2011-0499.)

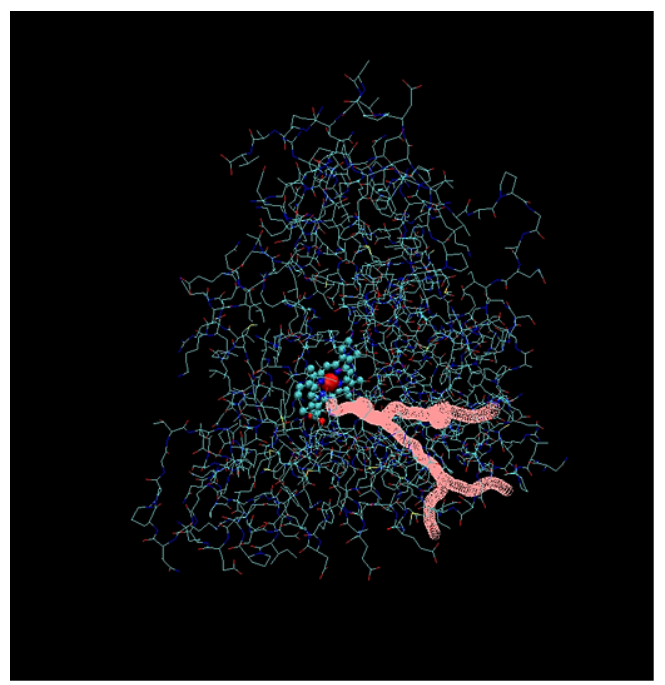

Fig. 15. Two groups of channels in pink identified by our method: up-horizontal: Channels D and G, down crosswise: channels $\mathrm{E}$ and $\mathrm{F}$ (details see Table 2, presentation as by Fig. 14). (Colors are visible in the online version of the article; http://dx.doi.org/ 10.3233/SPE-2011-0499.)

3. The longest channel of our study, B, should be analyzed together with the closely-related (and much shorter) channels F and E. These two channels were identified as group 2 of Caver's tunnels. However, B itself, although identified by our method as possible and having the largest-radius opening between $\alpha$ carbons, is less probable because of the necessity to pass through the complicated, more dispersed, and less hindered pathways near helix A and $\beta$-sheet 1.

4. The channel $\mathrm{C}$ was not identified by Caver method. It is interesting because it will allow the exit of the product near channels $\mathrm{F}$ and $\mathrm{G}$ and helix I. It is relatively narrow (4.1 $\mathrm{A}$ ). 
The final comparison of our group of channels enabled us to confirm their general agreement with both Poulos' channels and Caver's channels.

\section{Conclusion}

This work allowed us to elaborate two new, relatively simple methodologies to determine the access channels leading to the heme of the Cyt P450, by using the protein's PDB X-ray data even though this data was of low resolution. Both methodologies, based on the identification of "soft spots" within any protein structure, can be used on different proteins to find transport channels to reaction sites for substrates of given dimensions. The methods established in this work are as efficient as Caver or MolAxis, and faster than the previously-developed "slice" method. Caver and recent versions of MolAxis are more user-friendly than the methodologies described in this paper; however, this could easily be improved.

With the methodology established in this work, we can find many new channels fitting specific substrate sizes, and more strongly confirm the ones already found. This comparison will allow us to identify many new and unexplored channels with potential for use in, for example, in vitro enzyme analysis. Additionally, this confirms that these new methodologies are functional.

The a priori identification of channels leading to active sites of different biological enzymes is very important. The activity of enzymes such as Cyt P450 that are often implicated in drug metabolism can be further studied with the help of the identified channels. One such possibility is simply blocking the passage of the channels in vitro, and then examining the effect this has.

The findings of this study present a potential area of investigation regarding the entrance and exit of different kinds of substrates into the proteins, and will contribute to better understand the mechanisms of entry, an initial step for the transport of any kind of substrate via the channel and its extension into the cell membrane channels. The identification of what types of amino acids are close to the entry, for instance, could clarify the question of the way in which substrate translocation takes place. At the channel entry, the proximity of (for example) the acidic amino acids should be responsible for the orientation of the basic substrate into any given channel. At the other end of a channel, the Tyr 96 plays an active role during the oxidation process, assuming the necessary retention of substrate over the heme site and a necessary contact time for oxidation. ${ }^{3}$

For the test substrate of this study- $\beta$-carboline, an alkaloid of roughly steroidal dimensions for which we know how the CP450-promoted oxidation occurs, all channels found led to the region of oxidation via the relatively helix-poor domain near Tyr $96 .{ }^{4}$

The channels identified by all three methods described in this paper are certainly the largest ones responding to the substrate dimensions. For a carboline substrate, all five channels found via the spheres method were suitable for the substrate to enter, travel through, and reach the heme cavity where it was oxidized. $^{5}$

\footnotetext{
${ }^{3}$ As observed by Poulos [7,11,21,39,50,51], the specific space configuration of, e.g., camphor-oxidated iron heme and the pair of amino acids Cys 357 and Tyr 96, is necessary to achieve an oxidation.

${ }^{4}$ The Lipinski rules [2-6,14,22,33,40,45,47-49] suggest that the substrate molecules have a molecular weight less than about 500, have a calculated $\log P$ between -1 and +5 , and do not donate more than 5 hydrogen bonds nor accept more than $5 \times 2$ hydrogen bonds. This study is in perfect correlation with the Lipinski rules: the substrate we used had a molecular weight of 226 (well below 500) and had 3 hydrogen bonds.

${ }^{5}$ During the oxidation, the proteins could change their conformation in order to "choose" their "favourite" sites that facilitate exit from or entry into the cell. The change of conformation may represent another pathway by which large protein or enzyme molecules translocate channels greater than $2.44 \AA$ A. Furthermore, the presence of amino acids could possibly determine the charge of channels in which protein molecules go through.
} 
The identification of potential channels could also further support the hypothesis of substrate transport via the channels. However, caution is advised, despite many partial theories already advanced on this subject. ${ }^{6}$

The comparison of our three methodologies with the state-of-the-art (Caver) method used by Poulos in vivo (our methods confirm two channels found by Poulos) showed that the spheres method is very complete and precise, enabling the identification of even more potential channels in any given protein. ${ }^{7}$ As already stated, our two methods predicted the channels for transport of substrates of any size. In this respect, both methods are as versatile as Caver's (in which, however, the radius should be estimated when beginning the modeling) (Fig. 16).

The final validation of our sphere methodology was done using the results obtained via Poulos and Caver's methods. The comparison to these two studies showed that two main channels were found in our study and were confirmed via Caver's method. The third Caver's channel, however, was not found by our method. The three channels found in our study but not reported by Caver could be explained by our use of a reduced number of atom coordinates in our computation, as this sensitive gain of time for the calculations can sometimes lead to the identification of longer-than-usual channel lengths. At the same time, our results give more perspectives on the transport of larger substrates, allowing the rapid evaluation of possible pathways. This parameter has not yet been performed in Caver calculations where the exact diameters of channels are obtained. As such, our methodology is not only simpler and faster, but also takes into account the transport of many oversized substrates not allowed in Caver calculations. To do that, it is necessary to allow the dynamic openings of the passages of lesser diameter to larger substrates.

In this respect, our method has a complementary role in a complete analysis of possibilities for substrate oxidation by CP450 enzymes.

\footnotetext{
${ }^{6}$ Transport of carboline (1) via channels found in this study. The hypothesis of transport of basic indolic substrate via channels should be related to the presence of acidic amino acids such as Tyr, Asp or Glu within the channel. Within such a model, the number of these "buoys" on the route of the substrate through water-filled channels indicates that the formation of possible ionic bondings between the basic substrate and acidic amino acids should be responsible for the passive advancement of substrate via a channel. The $\mathrm{N}_{5}$ of $\mathbf{1}$ in particular is apt to form such associations. Its dissociation leads to the progressive advancement of substrate via the channel. This binding of the substrate to this functional group is weak and does not lead to permanent fixation of substrate on the channel side under the particular physiological conditions.

${ }^{7} \mathrm{~A}$ detailed comparison of our sphere methodology with the Caver methodology led to the following conclusions: both methodologies use spheres for the approximate estimation of the channel size, and in both cases the smallest connexion between two spheres of the channel gives the effective channel size.

However, in Caver's method, the channel starts from the cavity and finishes when both the previously requested number of channels and the outer surface of the protein are reached. Caver uses the Dijkstra algorithm for the search of shortest channels (MolAxis is based on the Voronoi diagram [50,51]). In our method, the large number of channels start at a certain distance from the cavity. The number of such starting points could be from one to many thousands. For each starting point we are seeking both the half-channel leading to the exit at the outer surface of the protein, and another half-channel leading to the heme (we are using the sphere instead of the convex surface). The sum of these two half-channels gives the full channel. After many such channels are calculated, they are selected according to the same exit was made (in practice, this means "the closest exits", within a reasonable limit of proximity, usually of the one having a fraction of Angstrom of distance). Finally, for each such group of channels, those with the largest radii are found and are considered as being representative to this group of channels.

For the representative channel the exact (true) exit point is found, which is closer to the inner site of the protein than the sphere which is limiting the outer surface. It is then possible that the true exit point is slightly deeper in the protein body than is the exit point calculated by Caver's method. Finally, in our case for the 1AKD P450 Cam (13) we found five (or a maximum of seven) channels, for the same protein where Caver's calculations, also gave five because only five channels were requested at the beginning of modeling. Is that the result of preliminary evaluation of the protein by the other methods, or the result of our expectation that CP450 would not have many channels? The simplest (and probably the only) way to solve this difficulty is then to compare the radii of the channels found and choose the largest ones from these groups.
} 

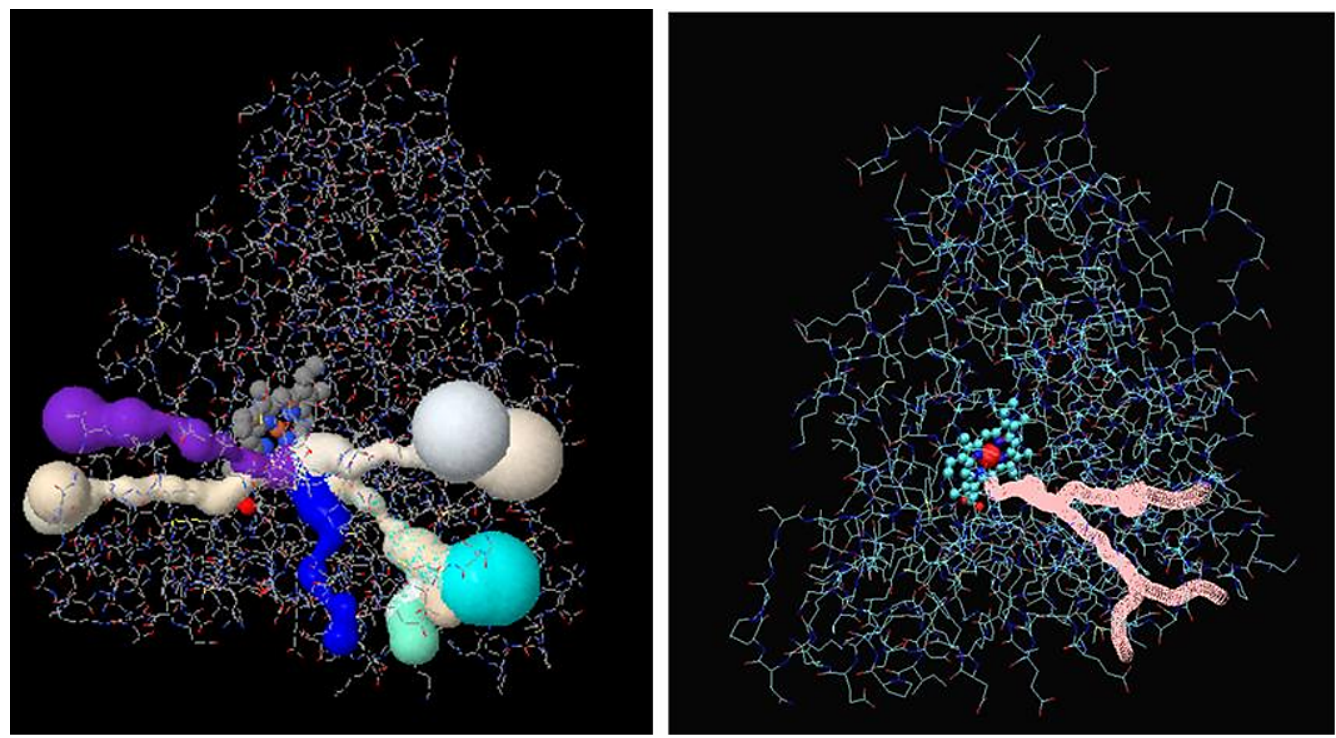

Fig. 16. Visual comparison of channels obtained by Caver-MolAxis (left) and our method (right) (see Cover). (Colors are visible in the online version of the article; http://dx.doi.org/10.3233/SPE-2011-0499.)

The model data used for these computations are reduced to the carbon backbone. Such limitations enabled better identification of the in vitro channels. However, any model of an a priori search for channels from the $\mathrm{X}$-ray data uses the in vitro reasoning, and is static in nature. The model developed in this study does not take into consideration the "pneumatic" nature of the protein and of the transport of the substrate through the channel. The diameter of the channel openings can decrease or increase following thermal modification of the protein - for instance, with the expansion of the channel during substrate transport.

In order to complete this study, this pneumatic effect should be considered. That effect is important for all modeled proteins since they are filled with water; such an expansible model could be constructed where even previously-discovered small-radius channels would be justified. These methods could also explore the channels on the dorsal part of the protein, in an in vitro context.

Finally, it will be necessary to improve the identification method for the channels with the spherical coordinates system and for those channels' entry points from the exterior of the protein, imitating the in vivo oxidation to make them more user-friendly, and thereby allowing faster comparison with channels identified by in vivo studies in particular.

This study also allowed us to validate the hypothesis of the oxidation mechanism, using some NMR data for both the starting carboline $\mathbf{1}$ and its hydroxylated derivatives (as shown in Appendix 3, Fig. 18 and Tables 3-5).

\section{Experimental}

\subsection{Computation}

Details of computation according to straight and curved channels search methodology are available on request from the corresponding author (C.K. Jankowski) or from [13] (Doucet thesis). 


\subsection{Method of spheres}

The detailed sphere method, as described in Appendix 2, is also available from the authors (J.B. Chiasson and C.K. Jankowski at the Université de Moncton).

\section{Acknowledgements}

We would like to acknowledge the participation in the preliminary step of this study of Professor Francisco Söler and Pierre J. Plourde. The helpful discussion with our colleagues from the Engineering School of the Université de Moncton, professors N.K. Srivastawa and N. Turkkan, was greatly appreciated. Our approach was strongly influenced by the civil engineering methodology of passing cables through the semi-hidden or shadowed openings in order to, e.g., reinforce partially-damaged structures. This technology enables for instance early structural damage detection under X-ray monitoring. We would like to thank the Université de Moncton FESR for constant support of our modeling projects.

\section{Appendix A. Slicing method}

This method was initially developed with help of Dr. F. Söler and P.J. Plourde. The method was inspired by the Lewis and Moereels study [31].

The data of the Cyt P450 were cut into 1- $\AA$-thick slides parallel to the heme surface and numbered from this origin. As a result, a total of 45 slices were established. The computer assisted comparison of two consecutive slices, e.g., $n$ and $n+1, \ldots$, enabled the determination of the possible channels, as a sequence of "holes" of given diameter through which the substrate when travelling toward the heme can pass, then be oxidized. All channels too small to allow the passage of the substrate were eliminated. In our case the carboline (1) substrate was considered as a $2 \AA \times 4 \AA \times 8 \AA$ rectangular box. Such a box was fitted through all possible openings via its smallest surface. The repetition of this operation to other $(n+1, n+2)$-type pairs of $1 \AA$ slices has led to the determination of several channels having suitable dimensions for the passage of the alkaloid but which miss the heme target (channel A). However, two channels B and C do reach the heme. Channel B leads to the heme without going through the entire Cyt P450 body; and only channel C enters the heme region by going through the whole Cyt P450 body.

\section{A.1. Channels identified by the "slicing" method (Fig. 17)}

List of key amino acids: 179 Y Tyr, 245 D Asp, 395 I Ile, 171 E Glu, 172 E Glu, 181 T Thr, 188 D Asp, 170 Y Tyr F, 316 D Asp.

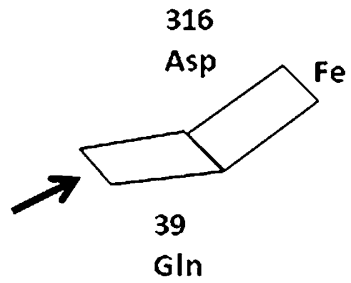

A

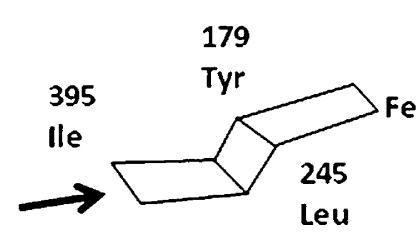

B

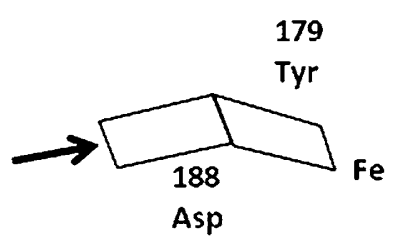

C

Fig. 17. Channels A, B and C as identified by slicing method [12,13,25]. 


\section{A.1.1. Channel description}

Channel A: between $\beta 5$ and helix I (under helix F toward Fe) key amino acids: Tyr 179, Asp 245, Ile 395 limited by Gln 39, Asp 316 and heme Fe.

Channel B: between $\beta 3$ and helix F. Key amino acids Tyr 179, Thr 188, Ile 395 and Leu 245.

Channel C: between $\beta 1$ and helix A. Key amino acids: Tyr 179, Asp 188, close to heme Fe.

The main Poulos channel is localized between $\beta 5$ and helices F and B. Key amino acids are Thr 185, Phe 87 and Ile 395.

The comparison of channels B and C led to the conclusion that channel B is larger, but longer than C. If the substrate is travelling through the channel $\mathrm{B}$, then the substrate could be oxidized, giving the observed beta-hydroxylation of all three methylenes, but the molecule should then travel backward to the exit via the same channel. The hydroxylated metabolite's eventual backward exit is the most puzzling result obtained in this work.

Several other B- and C-type channels were eliminated from consideration because they would lead the substrate to the heme area beneath the porphyrin plane, which would not result in the observed stereochemistry of the metabolites, and would affect the stereo or regioselectivity of the oxidation process.

The volume of the heme pocket allows the carboline (or other molecule which is at most steroid-size) to rotate around its own axis, but not to travel to the opposite side of the heme. (Cannot reach the ferric oxygen of heme and further rotate within a limited space of the cavity.)

The specific $\beta$-oxidation of some keto steroids on the methylene C-11 and methyl C-18 (by Cyt P450 $11 \beta, 18 \beta$ ) should also be explained similarly $[9,18,24]$.

From the selection of channels found by the slicing methodology, channel $\mathrm{C}$ seems to be the most probable channel for oxidation of the carboline because its stereochemistry matches that of the hydroxylated metabolites, and because they exit the heme cavity more easily. It is worth noting that channel C corresponded to the one identified by Poulos [41-43].

\section{Appendix B. Algorithm for sphere method}

\section{B.1. Computing the intersection between two cavities}

Cavities A and B have radius $r_{\mathrm{A}}$ and $r_{\mathrm{B}}$, respectively. The distance between their centres is $d$. There are four cases:

(a) There is no intersection between the two cavities if $r_{\mathrm{A}}+r_{\mathrm{B}}<d$. In such a case, no path can exist between those two cavities.

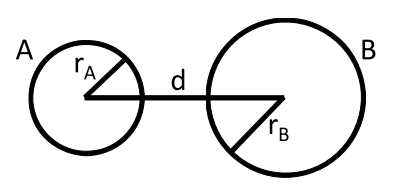

(b) There is inclusion if either $r_{\mathrm{A}}+d \leqslant r_{\mathrm{B}}$ and $r_{\mathrm{B}}>d$ or $r_{\mathrm{B}}+d \leqslant r_{\mathrm{A}}$ and $r_{\mathrm{A}}>d$. In particular, this can happen when both cavities are bounded by the same atom. In this case, there is a path between $\mathrm{A}$ and $\mathrm{B}$. The size of the path is limited by the radius of the smaller cavity. 


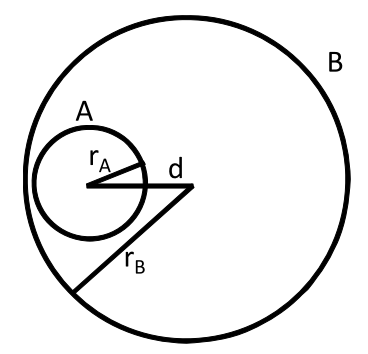

(c) There is intersection, but not between the two centres, if either $r_{\mathrm{A}}+d>r_{\mathrm{B}}$ and $r_{\mathrm{B}}>d$ or $r_{\mathrm{B}}+d>r_{\mathrm{A}}$ and $r_{\mathrm{A}}>d$. In this case, because the smaller cavity is mostly included inside the larger cavity, the radius of the connection between the two cavities is the radius of the smaller cavity.

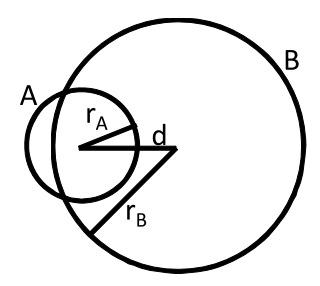

(d) Otherwise, the intersection is between the two centres if $r_{\mathrm{A}}+r_{\mathrm{B}}>d$ and $r_{\mathrm{A}}<d$ and $r_{\mathrm{B}}<d$. In such a case, a formula based on right-angle triangles and similar triangles properties was used to compute the radius of the intersecting circle between the two spherical cavities. The radius $r$ of that circle is the radius of the connection between the two cavities.

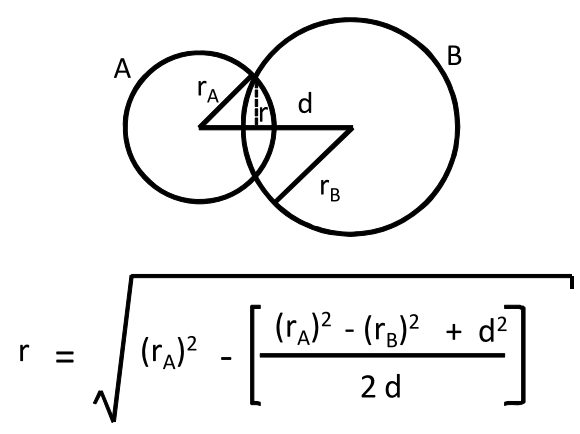

\section{Appendix C}

\section{C.1. NMR study of carboline (1)}

The NMR (proton and carbon-13) polydimensional studies of carboline were performed in order to compare the behavior of an aza-tetracyclic skeleton behaviour within a heme cavity to the behavior of a steroidal skeleton in the same location (relative to oxidation with CP450). This was realized by selective deuteration of carboline (1), already reported by our group $[14,22,33,48]$ on the methylenes C-4, C- 6 and 
C-7. The essential question was the conformation adopted by the carboline (in chloroformic solution) and, as a consequence of this, the approach to the heme- area which could be identical or the opposite to the steroid approach.

It is already known that the oxidation of several steroids with CP450 leads to the hydroxylation of methylenes or methines - for example, of methylenes at C-11 or C-12 (as well as the methine C-17 or methylene $\mathrm{C}-3$, etc.) [31]. This supposes that the steroid, with both $\mathrm{A} / \mathrm{B}$ rings and $\mathrm{C} / \mathrm{D}$ rings junctions trans, means with $\mathrm{H}-6 \alpha$ and $\mathrm{H}-14 \alpha$ hydrogens, and $\mathrm{C}-18$ and $\mathrm{C}-19$ methyls being $\beta$ oriented, enters the cavity with the $\mathrm{C}$ and $\mathrm{D}$ rings forward. The oxidation of $\mathbf{1}$ with CP450 in our previous studies confirmed that the alicyclic rings $\mathrm{C}$ and $\mathrm{D}$ of several alkaloids, carboline (1) included, should enter the cavity presenting the alicyclic end to oxidation by $\mathrm{Fe}-\mathrm{O}_{2}$ complex.

Careful examination of NMR spectra of carboline (1) confirmed that the proton $12 \mathrm{~b}$ is $\beta$-oriented (on the trans junction of the $C$ and $D$ rings), as witnessed by the neighbouring $C-12 b$ at $60.26 \mathrm{ppm}, \mathrm{C}-6$ at $53.55 \mathrm{ppm}$ and C-4 at $55.71 \mathrm{ppm}$ chemical shifts. These results are in agreement with Gribble's studies of indolic alkaloids and their specifically deuterated analogs [16,17,29].

These enabled us to advance the hypothesis that, if the junction of two rings is trans $(\mathrm{H}-12 \mathrm{~b}$ toward the nitrogen lone pair), then the carboline conformation is similar in this respect to the steroidal one.

The details of NMR analysis are presented in the following three tables: Table 3 has detailed identification of $\mathrm{H}-1$ and $\mathrm{C}-13$ signals, Table 4 has long-range correlation $\mathrm{H}-1-\mathrm{C}-13$ and Table 5 has NOE data, which is essential in the conformational studies in order to corroborate the $\delta$-oxidation mechanism hypothesis.

Table 3

Identification of $\mathrm{H}-1$ and $\mathrm{C}-13$ resonances for $1(\mathrm{CDCl} 3)$ in $\delta(\mathrm{ppm})$ (as recorded on Bruker AC, $300 \mathrm{MHz}$ )

\begin{tabular}{cccccc}
\hline Position & $\delta_{\mathrm{C}-13}(\mathrm{ppm})$ & Mult & $\delta_{\mathrm{H}-1}(\mathrm{ppm})$ & & \\
\hline 1 & 29.98 & $\mathrm{~S}$ & 2.07 & $\mathrm{eq}$ & \\
& & & 1.61 & $\mathrm{ax}$ & \\
2 & 24.29 & $\mathrm{~S}$ & 1.91 & $\mathrm{eq}$ & \\
& & & 1.51 & $\mathrm{ax}$ & \\
3 & 25.71 & $\mathrm{~S}$ & 1.74 & - & \\
4 & 55.71 & $\mathrm{~S}$ & 3.03 & - & \\
& & & 2.39 & & \\
6 & 53.55 & $\mathrm{~S}$ & 3.08 & $\mathrm{eq}$ & \\
& & & 2.65 & $\mathrm{ax}$ & \\
7 & 21.55 & $\mathrm{~S}$ & 3.01 & $\mathrm{ax}$ & \\
& & & 2.70 & $\mathrm{eq}$ & \\
$7 \mathrm{a}$ & 108.14 & $\mathrm{q}$ & - & - & \\
$7 \mathrm{~b}$ & 127.51 & $\mathrm{q}$ & - & - & \\
8 & 118.12 & $\mathrm{t}$ & 7.47 & - & $\mathrm{ddd}$ \\
9 & 119.39 & $\mathrm{t}$ & 7.08 & - & $\mathrm{dt}$ \\
10 & 121.30 & $\mathrm{t}$ & 7.13 & - & $\mathrm{dt}$ \\
11 & 110.71 & $\mathrm{t}$ & 7.30 & - & $\mathrm{ddd}$ \\
$11 \mathrm{a}$ & 135.97 & $\mathrm{q}$ & - & - & - \\
12 & - & & 7.70 & - & $\mathrm{br} . \mathrm{s}$ \\
$12 \mathrm{a}$ & 135.04 & $\mathrm{q}$ & - & - & - \\
$12 \mathrm{~b}$ & 60.23 & $\mathrm{t}$ & 3.25 & Ax & - \\
\hline
\end{tabular}


Table 4

Long range $\mathrm{H}-1 / \mathrm{C}-13$ correlations

\begin{tabular}{|c|c|c|c|}
\hline$\overline{\delta_{\mathrm{H}-1}(\mathrm{ppm})}$ & $\delta_{\mathrm{C}-13}(\mathrm{ppm})$ & & \\
\hline 7.47 & 108.14 & (7a) & ${ }^{3} \mathrm{~J}$ \\
\hline \multirow[t]{2}{*}{ (8) } & 121.30 & (10) & ${ }^{3} \mathrm{~J}$ \\
\hline & 135.97 & (11a) & ${ }^{3} \mathrm{~J}$ \\
\hline 7.30 & 119.39 & (9) & ${ }^{3} \mathbf{J}$ \\
\hline (11) & 127.51 & (7a) & ${ }^{3} \mathrm{~J}$ \\
\hline 7.13 & 118.12 & (8) & ${ }^{3} \mathrm{~J}$ \\
\hline (10) & 138.97 & (11a) & ${ }^{3} \mathrm{~J}$ \\
\hline 7.08 & 110.71 & $(11)$ & ${ }^{3} \mathrm{~J}$ \\
\hline (9) & 127.51 & (7b) & ${ }^{3} \mathbf{J}$ \\
\hline $3.0-3.1$ & 21.55 & (7) & ${ }^{3} \mathrm{~J}$ with (6eq), $\left[{ }^{4} \mathrm{~J}\right.$ with (4eq) $]$ \\
\hline$(7 \mathrm{ax})$ & 24.29 & (2) & ${ }^{3} \mathrm{~J}$ with (4eq), \\
\hline (6eq) & 60.23 & $(12 b)$ & ${ }^{3} \mathrm{~J}$ with (6eq),${ }^{3} \mathrm{~J}$ with (4eq), $\left[{ }^{4} \mathrm{~J}\right.$ with $\left.(7 \mathrm{ax})\right]$ \\
\hline$(4 \mathrm{eq})$ & 108.14 & (7a) & ${ }^{3} \mathrm{~J}$ with (6eq) ${ }^{2} \mathrm{~J}$ with (7ax) \\
\hline $2.6-2.7$ & 135.04 & (12a) & ${ }^{3} \mathrm{~J}$ with $(7 \mathrm{eq})$ \\
\hline$(7 \mathrm{eq})$ & 60.23 & $(12 b)$ & ${ }^{3} \mathbf{J}$ with $(6 a x),\left[{ }^{4} \mathbf{J}\right.$ with (7eq) $]$ \\
\hline$(6 a x)$ & 108.14 & (7a) & ${ }^{3} \mathbf{J}$ with $(6 \mathrm{ax}),\left[{ }^{2} \mathrm{~J}\right.$ with (7eq) $]$ \\
\hline 2.39 & 60.23 & $(12 b)$ & ${ }^{3} \mathbf{J}$ \\
\hline$(4 a x)$ & & & \\
\hline 2.07 & 25.71 & (3) & ${ }^{3} \mathbf{J}$ \\
\hline (1eq) & & & \\
\hline
\end{tabular}

Table 5

Selected NOE effects for carboline (1)

\begin{tabular}{lccc}
\hline Irradiated proton & Observed NOE at & $(*)$ & Identification of protons \\
\hline $4 \mathrm{ax}$ & 3.03 & $(\mathrm{~s})$ & $4 \mathrm{eq}$ \\
& 3.25 & $(\mathrm{w})$ & $12 \mathrm{~b} \mathrm{ax}$ \\
& 1.70 & $(\mathrm{w})$ & $3(?)$ \\
$1 \mathrm{eq}$ & 1.61 & $(\mathrm{~m})$ & $1 \mathrm{ax}$ \\
& 3.25 & $(\mathrm{w})$ & $12 \mathrm{~b} \mathrm{ax}$ \\
$\mathrm{eq}$ & 1.51 & $(\mathrm{~m})$ & $2 \mathrm{ax}$ \\
$12 \mathrm{~b}$ ax & 2.39 & $(\mathrm{~m})$ & $4 \mathrm{ax}$ \\
& 2.65 & $(\mathrm{~m})$ & $6 \mathrm{ax}$ \\
& 2.07 & $(\mathrm{w})$ & $1 \mathrm{eq}$ \\
\hline
\end{tabular}

Notes: $(*) \mathrm{s}-$ strong, $\mathrm{m}$ - medium, $\mathrm{w}$ - weak.

Some conclusions reached from these data lead to the hypothesis of the entry of $\mathbf{1}$ into the cavity, as presented in Fig. 18. As far as the conformation of the carboline is concerned, it could then be assumed that it is similar to that of steroidal molecules, for example corticosterones.

The NOE data for original substrate 1 showed the $\mathrm{H}-12 \mathrm{~b}$ proton with NOE effect to protons $\mathrm{H}-4 \mathrm{ax}$, $\mathrm{H}-6 \mathrm{ax}$ (as well as to $\mathrm{H}-1$ eq; however, the $\mathrm{H}-7$ protons are joined into multiplets, which are difficult to resolve at $300 \mathrm{MHz}$ ). In the case of 1 and its three bi-deuterated derivatives $d_{2}-6,6, d_{2}-7,7$ and $d_{2}-4,4$, the NOE effect is absent on these two methylenes when oxidized with CP450. This observation could be interpreted as a replacement of the axial hydrogen by hydroxyl group (that is, oxidation of the axial 


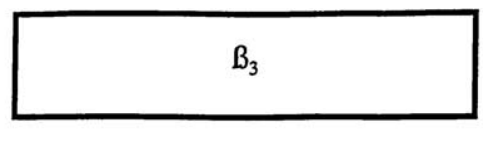

- V295

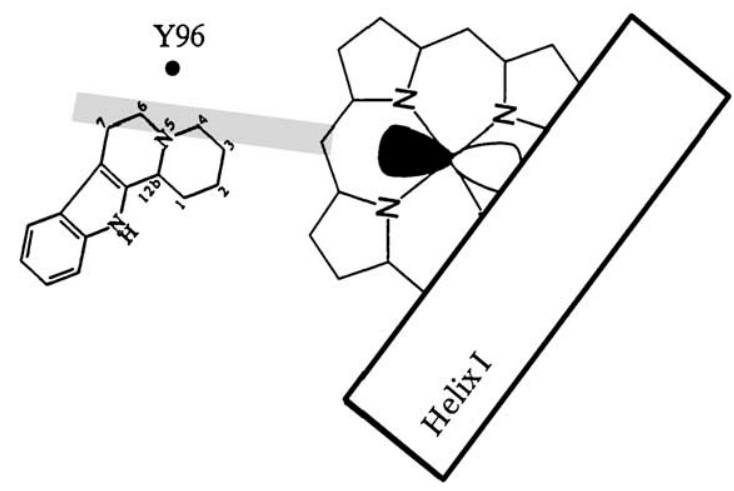

Fig. 18. Hypothesis of hydroxylation of methylenes C-4, C-6 and C-7 of carboline on heme.

hydrogen), leaving only the equatorial hydrogen, and in such a manner did not produce an important NOE effect to the axial $\mathrm{H}-12 \mathrm{~b}$ proton.

Additionally, this result confirmed the hypothesis of the entry of carboline alkaloids, similarly to steroids, by the $\mathrm{C}$ and $\mathrm{D}$ ring into the heme cavity and presenting the opposite to steroidal angular methyl side toward the heme (as presented in Fig. 18).

Despite the low recovery rate of hydroxylated carbolines (via extraction), this observation is useful for interpretation of the spectra of more complex bio-molecules. The NMR techniques are confirmed to be a valuable complement to molecular modeling in such analyses.

\section{References}

[1] 1AKD: pdB of P450 cam.

[2] 3D structure of CytP450 cam (PDB 2 CPP), available at: http://www.rcsb.org/pdb, May 16, 1997.

[3] D.H.R. Barton, Aldrichimica Acta 23 (1990), 3-12.

[4] D.H.R. Barton, J. Boivin, W.B. Motherwell, N. Ozbalik, K. Schwartzentruber and C.K. Jankowski, Nouv. J. Chem. 10 (1986), 387-398.

[5] D.H.R. Barton and D. Doller, Stud. Surf. Sci. Catalysis 66 (1991), 1-10.

[6] D.H.R. Barton and D. Doller, Acc. Chem. Res. 25 (1992), 504-512.

[7] P. Benes, P. Medek and J. Sochor, in: Proceedings Conference Visual Computing for Biomedicine (VCBM), 2008.

[8] T.H. Cormen, C.E. Leiserson, R.L. Rivest and C. Stein, in: Introduction à l'algorithmique, 2nd edn, MIT Press and McGraw-Hill, Cambridge and New York, 2001, pp. 595-601.

[9] M.A. Correia, in: Cytochrome P450, Structure, Mechanism and Biochemistry, P.R. Ortiz de Montellano, ed., Plenum Press, New York, 1995, pp. 607-630, Appendix B.

[10] J.R. Cupp-Vickery and T.L. Poulos, Nature Struct. Biol. 2 (1995), 144-153.

[11] J. Damborsky, M. Petrek, P. Banas and M. Otyepka, Biotech. J. 2 (2007), 62-67.

[12] G. Demengel and J.P. Pouget, Modèles de Bézier, des B-splines et des NURBS, Ellipses, Paris, 1998.

[13] C.K. Doucet, Thesis 4093, Étude du complexe Cyt P450, Université de Moncton, April 2005.

[14] F.A. Fares and C.K. Jankowski, Heterocycles 34 (1992), 2109-2116.

[15] R. Fedorov, D. Gosh and I. Schlihting, Science 287 (2000), 1615-1622.

[16] G.W. Gribble, Procedures J. Org. Chem. 37(11) (1972), 1833-1835.

[17] G.W. Gribble and R.B. Nelson, J. Org. Chem. 39 (1974), 1845-1850. 
[18] P.W. Harvey and D.J. Everett, J. Appl. Tox. 23(2) (2003), 81-87.

[19] C.A. Hasemann, R.G. Kurumbail, S.S. Boddupalli, J.A. Peterson and J. Deisenhofer, Structures 3(1) (1995), 41-62.

[20] C.A. Hasseman, K.G. Ravishandran, J.A. Peterson and J. Deisenhofer, J. Mol. Biol. 236 (1994), 1169-1185.

[21] http://bioinfo3d.cstau.ac.il/MolAxis/molaxis.html.

[22] C.K. Jankowski, M. Delaforge, M. Jaouen and H. Virelizier, Spectroscopy 13 (1997), 163-174.

[23] E.F. Johnson, Drug Metab. Disposition 31 (2003), 1932-1948.

[24] N. Kagawa and M.R. Waterman, in: Cytochrome P450, Structure, Mechanism and Biochemistry, P.R. Ortiz de Montellano, ed., Plenum Press, New York, 1995, pp. 419-442.

[25] D. Knuth, in: Metafont: The Program, Addison-Wesley, New York, 1986, pp. 123-131.

[26] S. Kominami, A. Higouchio and S. Takemori, Bioch. Bioph. Acta 937 (1988), 177-183.

[27] K. Konishi, K.-I. Oda, K. Nishida, T. Aida and S. Inoue, J. Am. Chem. Soc. 114 (1992), 1313-1317.

[28] D.F.V. Levis and H. Moereels, J. Comp. Aid. Mol. Design 6 (1992), 235-252.

[29] G.C. Levy, R.L. Lichter and G.L. Nelson, in: Carbon-13 Nuclear Magnetic Resonance Spectroscopy, 2nd edn, Wiley, New York, 1980, pp. 255-295.

[30] D.F.V. Lewis, C. Ioannides and D.V. Parke, Chem. Biol. Interactions 649 (1987), 39-60.

[31] D.F.V. Lewis and H. Moereels, J. Comp. Aid. Mol. Design 6 (1992), 235-252.

[32] C.A. Lipinski, F. Lomardo, B.W. Dominy and P.J. Feeney, Adv. Drug Delivery Rev. 23 (1997), 3-30.

[33] D.W. Lu and C.K. Jankowski, Spectroscopy 11 (1993), 59-78.

[34] D. Mansuy, Pure Applied Chem. 59 (1987), 759-770.

[35] D. Mansuy, P. Battoni and J.-P. Battoni, Europ. J. Bioch. 184 (1989), 267-283.

[36] T.J. McMurry and J.T. Groves, in: Cytochrome P450 Structure, Mechanism and Biochemistry, P. Ortiz de Montellano, ed., Plenum Press, New York, 1985, Chapter 1.

[37] C.A. Ouzonis and W.T. Melvin, Eur. J. Biochem. 198 (1991), 307-315.

[38] S.Y. Park, H. Shimatzu, S. Adachi, A. Nakagawa, I. Tanaka, K. Nakahara, H. Shoun, E. Obayashi, H. Nakamura, T. Iizuka and Y. Shiro, Nature Struc. Biol. 4 (1997), 827-832.

[39] M. Petrek, P. Banas, M. Otyepka, P. Kosinova, J. Koca and J. Damborsky, BMC Informatics 7 (2006), 316-324.

[40] T. Poulos and J. Kraut, J. Biol. Chem. 255 (1980), 8199-8205.

[41] T.L. Poulos, Nature 339 (1989), 580-581.

[42] T.L. Poulos, B.C. Finzel, I.C. Gunsalus, G.C. Wagner and J. Kraut, J. Biol. Chem. 260 (1985), 16122-16130.

[43] T.L. Poulos, B.C. Finzel and A.J. Howard, J. Mol. Biol. 195 (1987), 687-700.

[44] T.L. Poulos and A.J. Howard, Biochemistry 26 (1987), 8165-8174.

[45] T.L. Poulos, M. Perez and G.C. Wagner, J. Biol. Chem. 257 (1982), 10427-10429.

[46] K.G. Ravichandran, S.S. Boddupalli, C.A. Hasemann, J.A. Peterson and J. Deisenhofer, Science 261 (1974), 4037-4040.

[47] C. Sheu, S.A. Richert, P. Cofré, B. Ross, A. Sobkowiak and D.T. Sawyer, J. Am. Chem. Soc. 112 (1990), $1936-1942$.

[48] J. Sopkova, J.C. Smith, M. Delaforge, H. Virelizier and C.K. Jankowski, Eur. J. Biochem. 251 (1998), 398-404.

[49] M.R. Waterman and E.F. Johnson, Cytochrome P450, in: Methods in Enzymology, Vol. 206, Academic Press, 1991.

[50] E. Yaffe, D. Fishelovitch, H.J. Wolfson, D. Halperin and R. Nussinov, Proteins: Structure and Bioinformatics 73 (2008), $72-86$.

[51] E. Yaffe, D. Fiselovitch, H.J. Wolfson, D. Halpern and R. Nussinov, Nucl. Acid Res. 36 (2008), W210-W215.

For Barton's Gif oxidation see [3-6].

For Fe-picolinate oxidations see [47].

For cytochrome P450 camphor complex Putida pseudomonas see [45].

For bacterial CP450 see $[19,27,28,30,34-36,38,44,46]$.

For Bézier algorithms see [12,13,25].

For Caver (MolAxis) online service see [7,11,21,39,50,51].

For tunnels (channels) see [7].

For MolAxis description see [21].

For Voronoi algorithm and diagram see [50,51].

For membrane CP450 see [26,37].

For X-rays for this modeling see [15]. 


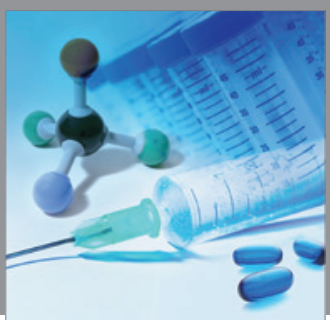

International Journal of

Medicinal Chemistry

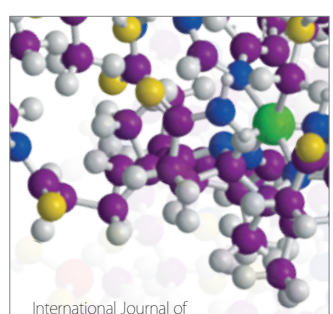

Carbohydrate Chemistry

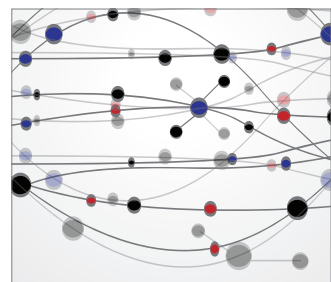

The Scientific World Journal
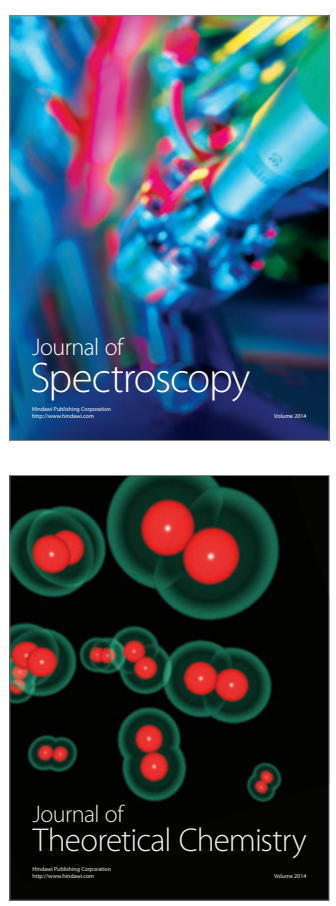
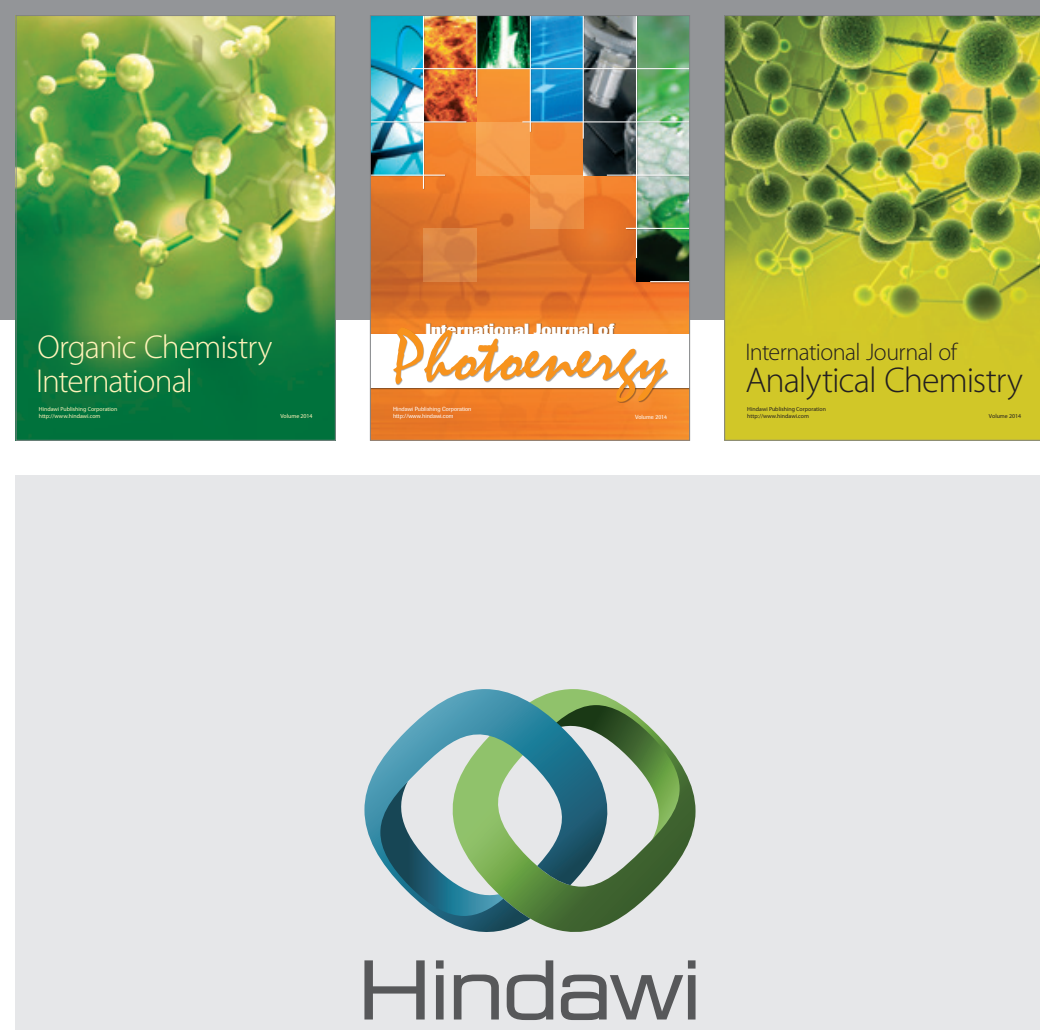

Submit your manuscripts at

http://www.hindawi.com
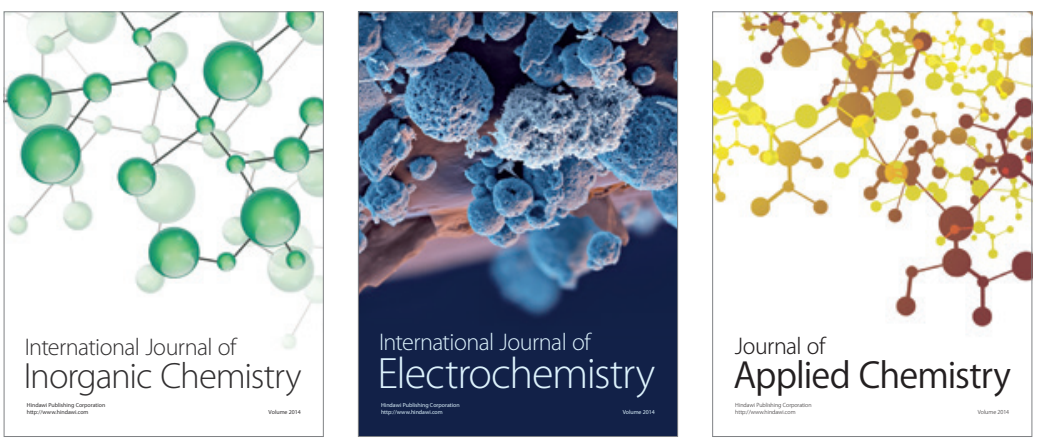

Journal of

Applied Chemistry
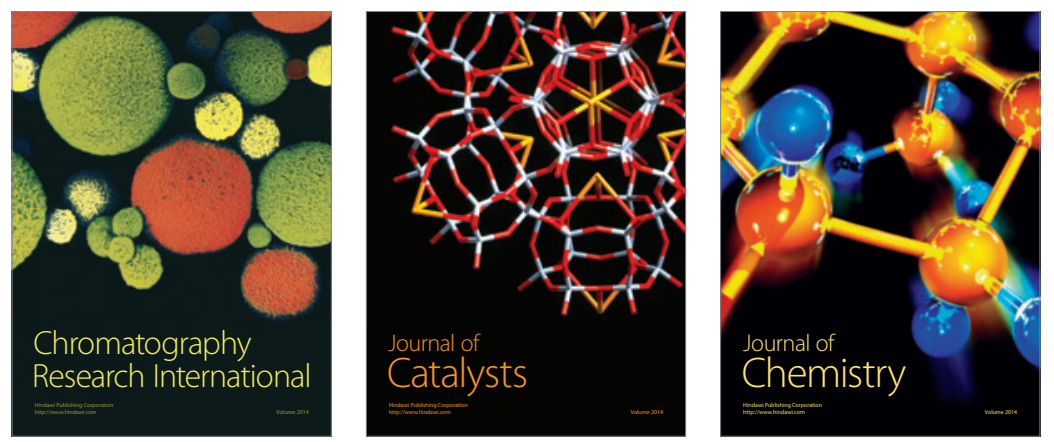
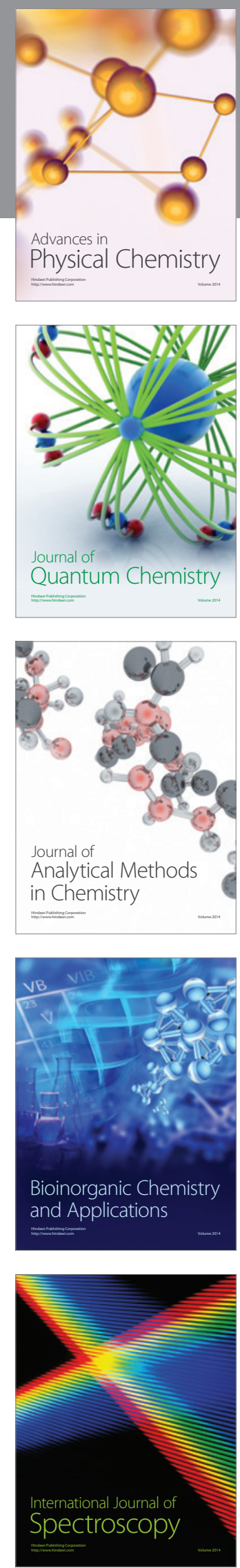